APS

physics

This is the accepted manuscript made available via CHORUS. The article has been published as:

\title{
High-spin proton alignments and evidence for a second band with enhanced deformation in $\wedge\{171\} \mathrm{Hf}$
}

Y. C. Zhang, Q. A. Ijaz, W. C. Ma, G. B. Hagemann, M. P. Carpenter, P. Chowdhury, D. M. Cullen, M. K. Djongolov, D. J. Hartley, R. V. F. Janssens, T. L. Khoo, F. G. Kondev, T. Lauritsen, E. Ngiojoi-Yogo, S. Ødegård, L. L. Riedinger, S. V. Rigby, D. G. Roux, D. T. Scholes, R. B. Yadav, and S. Zhu

Phys. Rev. C 85, 064307 - Published 7 June 2012 DOI: 10.1103/PhysRevC.85.064307 


\title{
High-spin proton alignments and evidence for second band with enhanced deformation in ${ }^{171} \mathrm{Hf}$
}

Y. C. Zhang ${ }^{1}$, Q. A. Ijaz ${ }^{1}$, W. C. Ma ${ }^{1}$, G. B. Hagemann ${ }^{2}$, M. P. Carpenter ${ }^{3}$, P. Chowdhury ${ }^{4}$, D. M. Cullen ${ }^{5}$, M. K. Djongolov ${ }^{6}$, D. J. Hartley ${ }^{7}$, R. V. F. Janssens ${ }^{3}$, T. L. Khoo ${ }^{3}$, F. G. Kondev 8 , T. Lauritsen ${ }^{3}$, E. Ngiojoi-Yogo ${ }^{1}$, S. Ødegård ${ }^{9}$, L. L. Riedinger ${ }^{6}$, S. V. Rigby ${ }^{5}$, D. G. Roux ${ }^{1 *}$, D. T. Scholes ${ }^{5}$, R. B. Yadav $^{1}$, and S. Zhu ${ }^{3}$

${ }^{1}$ Department of Physics, Mississippi State University, Mississippi State, Mississippi 39762, USA

${ }^{2}$ The Niels Bohr Institute, Blegdamsvej 17, DK-2100 Copenhagen, Denmark

${ }^{3}$ Physics Division, Argonne National Laboratory, Argonne, Illinois 60439, USA

${ }^{4}$ Department of Physics, University of Massachusetts, Lowell, Massachusetts 01854, USA

${ }^{5}$ Schuster Laboratory, University of Manchester, Manchester M13 9PL, United Kingdom

${ }^{6}$ Department of Physics and Astronomy, University of Tennessee, Knoxville, Tennessee 37996, USA

${ }^{7}$ Department of Physics, United States Naval Academy, Annapolis, Maryland 21402, USA

${ }^{8}$ Nuclear Engineering Division, Argonne National Laboratory, Argonne, Illinois 60439, USA

${ }^{9}$ Department of Physics, University of Oslo, N-0316 Oslo, Norway

(Dated: May 25, 2012)

\begin{abstract}
High-spin properties of the nucleus ${ }^{171} \mathrm{Hf}$ were studied through the ${ }^{128} \mathrm{Te}\left({ }^{48} \mathrm{Ca}, 5 n\right)$ reaction. Previously known bands have been extended to significantly higher spins and four new bands have been extracted from these data. The results are discussed within the framework of the cranked shell model aided by a comparison with level structures in the neighboring nuclei. The band crossings at rotational frequencies $\sim 500 \mathrm{keV}$ are interpreted as caused by the alignments of $h_{11 / 2}$ and $h_{9 / 2}$ proton orbitals. Band ED2 exhibits an alignment pattern similar to that of band ED1 which was reported in a recent paper and proposed to be built upon a second potential energy minimum involving the deformation-driving proton $i_{13 / 2} \otimes h_{9 / 2}$ configuration. It is likely that band ED2 is also associated with a deformation enhanced with respect to that of the normal deformed structures. Further experimental investigation is needed to ascertain the nature of this band.
\end{abstract}

PACS numbers: 21.10.Re, 23.20.Lv, 25.70.-z, 27.70.+q

\section{INTRODUCTION}

Considerable progress has been made over the past decade in the investigation of stable triaxiality in nuclei. The wobbling motion, a characteristic excitation mode of triaxial nuclei [1], has been established in ${ }^{163,165,167} \mathrm{Lu}[2-5]$, possibly in ${ }_{71}^{161} \mathrm{Lu}[6]$, and very recently in ${ }_{73}^{167} \mathrm{Ta}$ [7]. Triaxial strongly deformed (TSD) structures have also been observed in several other neighboring nuclei of the mass $A \sim 160$ region, e.g., in ${ }^{168} \mathrm{Hf}[8,9]$. Cranking calculations employing the ULTIMATE CRANKER (UC) code [10-12] suggest that the TSD minima with deformation parameters $\left(\varepsilon_{2}, \gamma\right) \sim\left(0.4, \pm 20^{\circ}\right)$ in the potential energy surfaces are stabilized by large single-particle shell gaps associated with proton numbers $Z=71$ and 72 and neutron numbers $N=94$ and $97[13,14]$.

Surprisingly, a number of strongly deformed bands have been observed in the heavier $\mathrm{Hf}$ isotopes, ${ }^{170} \mathrm{Hf}[15],{ }^{171,172} \mathrm{Hf}$ [16], ${ }^{173,174} \mathrm{Hf}[17,18]$, and ${ }^{175} \mathrm{Hf}$ [19]. These findings raised questions about the validity of the predicted TSD shell gaps. In fact, the observations raised the question of whether these bands are associated with TSD shapes or not. Based on a systematic study of the rotational properties of these bands, and the calculations using the UC code as well as the cranked relativistic mean-field approach, these sequences were proposed to fall into two groups. The bands in the first group are likely built on the proton $i_{13 / 2} h_{9 / 2}$ configuration and are associated with near prolate shapes and deformations $\left(\varepsilon_{2} \sim 0.3\right)$ enhanced relative to the normal-deformed (ND) nuclear shapes $\left(\varepsilon_{2} \sim 0.22\right)$ characterizing the respective ground states. The bands in the second group are likely associated with superdeformed prolate shapes with little triaxiality, involving the $\pi i_{13 / 2}$ (proton), as well as the $\nu j_{15 / 2}$ (neutron) orbitals originating from above the $N=126$ spherical shell gap [16]. Consequently, the two groups of bands are labeled as ED and SD bands, respectively. The measured quadrupole moments of $Q_{t} \sim 9.5 e \mathrm{~b}$ for band ED1 in ${ }^{171} \mathrm{Hf}$ [20] and $\sim 14 e \mathrm{~b}$ for the SD bands in ${ }^{172} \mathrm{Hf}[20]$ and ${ }^{173-175} \mathrm{Hf}[18,19]$ strongly support the above assessments when compared to $Q_{t} \sim 6.2 e \mathrm{~b}$ for the ND band [521]1/2 in ${ }^{171} \mathrm{Hf}[21]$.

In addition to our recent publication on the strongly deformed bands in ${ }^{171,172} \mathrm{Hf}$ [16], an extensive study of the ND level structure of ${ }^{171} \mathrm{Hf}$ has also been carried out. Here, we report on the new results for the ND level scheme and on the identification of a possible second ED band in ${ }^{171} \mathrm{Hf}$. Previously known ND bands have now been

\footnotetext{
* Current address: Department of Physics and Electronics, Rhodes University, Grahamstown, South Africa
} 
extended to significantly higher spins. The proton alignment at a rotational frequency of $\hbar \omega \sim 0.5 \mathrm{MeV}$ can now be fully delineated and investigated. Three new ND bands were also identified. High-spin band crossings and possible intrinsic configurations for each band are discussed based on cranking calculations aided by a comparison with data from neighboring nuclei.

\section{EXPERIMENT AND DATA ANALYSIS}

High-spin states were populated in ${ }^{171} \mathrm{Hf}$ with the ${ }^{128} \mathrm{Te}\left({ }^{48} \mathrm{Ca}, 5 n\right)$ reaction at a beam energy of $209 \mathrm{MeV}$. The ${ }^{48} \mathrm{Ca}$ beam was provided by the ATLAS facility at Argonne National Laboratory. The target consisted of $\sim 0.5 \mathrm{mg} / \mathrm{cm}^{2}$ isotopically-enriched ${ }^{128} \mathrm{Te}$ foils with $0.5 \mathrm{mg} / \mathrm{cm}^{2} \mathrm{Au}$ layers in front of the Te and $0.05 \mathrm{mg} / \mathrm{cm}^{2} \mathrm{Au}$ evaporated on the back. A beam wobbling device and a target wheel were used to help with heat dissipation in the target, thus allowing a larger beam current $(\sim 2 \mathrm{pnA})$ to be deposited. Coincident $\gamma$ rays were measured using the Gammasphere array [22] which consisted of 100 Compton-suppressed Ge detectors at the time of the experiment. A data set of approximately $2.1 \times 10^{9}$ coincidence events with fold three and higher was collected.

In the off-line analysis, the data were sorted into a database where the $\gamma$-ray energies and detector identification were stored for each event. The RADWARE software package [23] was used to construct three-dimensional (cube) and four-dimensional (hypercube) histograms, and to analyze the $\gamma$-ray coincidence relationships. In addition, an analysis of directional correlation from oriented states (DCO ratios) [24] was performed on the data set in order to determine the multipolarity of the $\gamma$ rays. A number of gated DCO matrices, with detectors at $31^{\circ}, 37^{\circ}, 143^{\circ}, 148^{\circ}$ and $163^{\circ}$ along the x-axis, and detectors from $58^{\circ}$ through $122^{\circ}$ along the y-axis, were constructed from the database for this purpose. The technique was calibrated with transitions of known multipolarity. The extracted DCO ratios from E2gated spectra fall into two distinct groups centered around 1.0 and 0.6 for stretched quadrupole and dipole transitions, respectively. Further discussion of the technique, including different gating conditions and DCO ratios of unstretched transitions, can be found in our previous publication [25]. The parity assignments are based on the multipolarities of linking transitions between bands, as well as on coincidence relationships that may introduce important constraints. In some instances, the proposed configurations of the bands were also taken into consideration.

\section{LEVEL SCHEME}

The high-spin structures of the ${ }^{171} \mathrm{Hf}$ nucleus have been studied before by Dracoulis [26] and Cullen et al. [21, 27]. The proposed ${ }^{171} \mathrm{Hf}$ level scheme resulting from this work is presented in Figs. 1 and 2 . Each band is labeled by a name, except for the three strongest bands where, for convenience of discussion, each signature is explicitly labeled as E, F, G, H, A, and B together with the associated Nilsson orbitals. These signatures will be referred to as "bands"

loosely, e.g., "bands A and B", as is customary. Table I lists all level energies and $\gamma$ rays that are observed in this work, together with the transition intensities and DCO ratios. The previously known levels and $\gamma$ rays [21, 27] are also included in the table. The $\gamma$-ray intensities are measured relative to that of $522-\mathrm{keV}$ transition in band $\mathrm{A}$, which has an intensity of 70 .

All previously known bands have now been extended to higher spins. For example, band B has been extended from spin $63 / 2$ to $79 / 2$, and band $\mathrm{H}$ from $71 / 2$ to a tentative level of spin $95 / 2$. Eleven $E 1$ transitions have been identified depopulating levels between $21 / 2^{-}$and $43 / 2^{-}$in bands $\mathrm{G}$ and $\mathrm{H}$ and feeding bands $\mathrm{A}$ and B. The $\gamma$-ray energies of transitions above level $73 / 2$ in band $\mathrm{E}$ have been changed from the previous publication [21]. Coincidence spectra representative of bands $\mathrm{B}$ and $\mathrm{H}$ are displayed in Fig. 3. The two high-K bands HK1 and HK2 built on the $K^{\pi}=19 / 2^{+}$and $K^{\pi}=23^{-}$isomeric states, respectively, have been extended from spins $43 / 2$ and $47 / 2$ to $65 / 2$ and 59/2. Coincidence spectra of these two bands are presented in Fig. 4. The previously observed transitions in bands X1 and X3 have been placed differently in the level scheme. Four new bands, ED2, X2, X4, and MAB, have been established. Band ED1 has been reported in our recent publication [16] and will not be discussed further here. Coincidence spectra of bands ED2, X1 - X4, and MAB are found in Figs. 3 and 5.

\section{A. Bands X1 - X4}

Only three $\gamma$ rays at 870,831 , and $787 \mathrm{keV}$ were observed previously in band $\mathrm{X} 1$, and the $362-\mathrm{keV}$ transition connecting this sequence with band E was tentatively suggested to be an unstretched $E 2$ transition with $\Delta I=1$ [21]. In the current work, band X1 is seen from spin $33 / 2^{-}$to $81 / 2^{-}$. Twelve new linking transitions between bands X1 and E have been identified. The measured DCO ratios of the higher-energy 930.4-, 978.1- and 1039.9-keV $\gamma$ rays, and the lower-energy 356.7- and $362-\mathrm{keV} \gamma$ rays are all close to 1 , indicating that they are either stretched $E 2$ or 
unstretched dipole transitions. Obviously, they cannot all be of the $E 2$ type. The most probable scenario is that the higher-energy $\gamma$ rays are of stretched $E 2$ character, and that the lower-energy $\gamma$ rays are unstretched $M 1$, or $M 1 / E 2$ admixed transitions, leading to a parity assignment of $\pi=-$ for band X1. It should also be noted that an $M 1 / E 2$ admixed transition may, depending on the mixing ratio and level spins, have DCO ratios ranging between 0.2 and 1.3. If all the higher-energy and lower-energy linking transitions were of the $M 1 / E 2$ type, and accidentally had DCO ratios of 1 , the spins of band X1 would be $1 \hbar$ lower. However, such a scenario is highly unlikely. Therefore, the spins and the negative parity of band $\mathrm{X} 1$ can be considered firmly established.

Band X2 extends from $33 / 2^{+}$to $49 / 2^{+}$. The sequence is linked to the coupled bands A and B by nine depopulating transitions. The DCO ratios of the 898.8-, 916.5-, 994.8-, and 1055.1-keV $\gamma$ rays are consistent with a stretched E2 nature, the ratios of the $567.4-$ and $458.1-\mathrm{keV} \gamma$ rays are consistent with stretched $M 1$ transitions, and the ratio of the 388.2-keV $\gamma$ ray is consistent with an unstretched dipole transition. Thus, the positive parity and the spins of the band were determined unambiguously.

Previously, four $\gamma$ rays in band X3 were observed and the sequence was directly connected to the $45 / 2^{+}$level in band A [21]. Now, band X3 is seen to feed the 49/2+ level in band X2 through a 846.7-keV stretched E2 transition. Thus, band X3 has positive parity, and the level spins can be determined. This band is weaker than all other normal deformed bands. The intensities of the strongest $\gamma$ rays in the band, at 843.9 and $896.2 \mathrm{keV}$, are approximately 0.3 .

Band X4 consists of a short sequence of four weak $\gamma$ rays. It feeds bands X2 and X3 through 908- and 970-keV stretched E2 transitions. Therefore, the band has been assigned positive parity. When measuring the DCO ratios for the 908- and 909-keV transitions, the summed peak of the two transitions were used. The total intensity of the band is less than 1.0, as represented by the sum intensity of the $970-$ and $909-\mathrm{keV} \gamma$ rays.

\section{B. Band MAB}

With a maximum intensity of $\sim 1.9$, band MAB is slightly stronger than bands X3 and X4. It feeds band B. The 837.6-keV $\gamma$ ray is the strongest interband linking transition. Its DCO ratio of 0.66 (6) is consistent with a stretched dipole character. A negative parity is suggested for band MAB because the linking transition most likely has an $E 1$ multipolarity. An $M 1$ transition of such high energy would be expected to exhibit an $E 2$ admixture resulting in a larger DCO ratio. Furthermore, if the band has a positive parity, strong $E 2$ transitions should be expected from MAB to band A, and these are not present. Additional support of the spin/parity assignments comes from the proposed configuration for the band which will be discussed below. A very weak $1048.2-\mathrm{keV}$ interband transition is placed in the level scheme tentatively. There is a $641-\mathrm{keV} \gamma$ ray depopulating the $37 / 2^{-}$level of band MAB, followed by a $515-\mathrm{keV}$ transition that feeds the $35 / 2^{+}$state in band B. An accurate DCO ratio cannot be obtained for this $515-\mathrm{keV}$ $\gamma$ ray because it cannot be resolved from the very strong 516 -keV transition in band B. Thus, we tentatively suggest a spin $37 / 2$ for the level depopulated by the $515-\mathrm{keV} \gamma$ ray.

\section{Band ED2}

Band ED2 consists of a sequence of ten transitions. With an intensity of $\sim 0.14 \%$ relative to total population in this reaction channel, the band is about ten times weaker than band ED1. By gating on the band members, it is possible to observe the lower part of band E up to spin $37 / 2^{-}$, indicating that the band belongs to ${ }^{171} \mathrm{Hf}$ and feeds band E. Unfortunately, detailed decay pathways could not be established and, consequently, the level energies and spins and parity of the band could not be determined.

TABLE I: Gamma-ray energies $\left(E_{\gamma}\right)$, relative intensities $\left(I_{\gamma}\right)$, DCO ratios, and suggested multipolarities, together with spins/parities $\left(J_{i}^{\pi}\right)$ and energies $\left(E_{i}\right)$ of the depopulated states in ${ }^{171} \mathrm{Hf}$, measured in the present work.

\begin{tabular}{cccccc}
\hline \hline$J_{i}^{\pi}$ & $\mathrm{E}_{i}(\mathrm{keV})$ & $\mathrm{E}_{\gamma}(\mathrm{keV})^{a}$ & $I_{\gamma}^{b}$ & $\mathrm{R}_{D C O}$ & Assignment \\
\hline & Bands A \& B & & & & \\
$7 / 2^{+}$ & $0^{c}$ & & & & \\
$9 / 2^{+}$ & $61.9^{c}$ & $61.9^{c}$ & & & M1/E2 ${ }^{c}$ \\
$11 / 2^{+}$ & 145.9 & 145.9 & & & E2 \\
& & 84.1 & & & M1/E2 \\
$13 / 2^{+}$ & 244.9 & 183.0 & $12(1)$ & $1.03(5)$ & E2 \\
& & 98.9 & $8.9(4)$ & $0.46(10)$ & M1/E2 \\
$15 / 2^{+}$ & 382.4 & 236.5 & $30(2)$ & & E2 \\
\hline
\end{tabular}


TABLE I (Continued.)

\begin{tabular}{|c|c|c|c|c|c|}
\hline$J_{i}^{\pi}$ & $\mathrm{E}_{i}(\mathrm{keV})$ & $\mathrm{E}_{\gamma}(\mathrm{keV})^{a}$ & $I_{\gamma}^{b}$ & $\mathrm{R}_{D C O}$ & Assignment \\
\hline & & 137.2 & $16(2)$ & $0.60(6)$ & $M 1 / E 2$ \\
\hline \multirow[t]{2}{*}{$17 / 2^{+}$} & 512.2 & 267.3 & $50(1)$ & $1.05(5)$ & E2 \\
\hline & & 129.8 & $11(2)$ & $0.5(1)$ & $M 1 / E^{2}$ \\
\hline \multirow[t]{2}{*}{$19 / 2^{+}$} & 716.4 & 334.0 & $40(1)$ & & E2 \\
\hline & & 203.6 & $16(2)$ & $0.53(10)$ & $M 1 / E_{2}$ \\
\hline \multirow[t]{2}{*}{$21 / 2^{+}$} & 866.3 & 354.1 & $53(2)$ & & E2 \\
\hline & & 150.2 & $18(2)$ & $0.45(12)$ & $M 1 / E_{2}$ \\
\hline \multirow[t]{2}{*}{$23 / 2^{+}$} & 1145.7 & 429.3 & $52(2)$ & & E2 \\
\hline & & 279.3 & $10(1)$ & $0.5(1)$ & $M 1 / E_{2}$ \\
\hline \multirow[t]{2}{*}{$25 / 2^{+}$} & 1306.3 & 440.0 & $68(2)$ & $1.2(1)$ & E2 \\
\hline & & 160.3 & $2.9(5)$ & $0.65(10)$ & $M 1 / E_{2}$ \\
\hline \multirow[t]{2}{*}{$27 / 2^{+}$} & 1661.6 & 515.9 & $53(1)$ & & E2 \\
\hline & & 354.9 & $8.5(7)$ & & $M 1 / E_{2}$ \\
\hline \multirow[t]{2}{*}{$29 / 2^{+}$} & 1828.1 & 521.8 & $70(5)$ & $1.08(5)$ & E2 \\
\hline & & 165.4 & $1.5(9)$ & & $M 1 / E 2$ \\
\hline \multirow[t]{2}{*}{$31 / 2^{+}$} & 2254.6 & 593.0 & $29(2)$ & & E2 \\
\hline & & 426.7 & $3.8(6)$ & & $M 1 / E_{2}$ \\
\hline \multirow[t]{2}{*}{$33 / 2^{+}$} & 2426.2 & 598.1 & $69(2)$ & & E2 \\
\hline & & 170.8 & $0.5(1)$ & & $M 1 / E 2$ \\
\hline \multirow[t]{2}{*}{$35 / 2^{+}$} & 2914.0 & 659.4 & $29(2)$ & & E2 \\
\hline & & 487.5 & $4.5(8)$ & & $M 1 / E 2$ \\
\hline \multirow[t]{2}{*}{$37 / 2^{+}$} & 3092.9 & 666.7 & $38(1)$ & & E2 \\
\hline & & 179.2 & $0.4(1)$ & & $M 1 / E 2$ \\
\hline \multirow[t]{2}{*}{$39 / 2^{+}$} & 3629.7 & 715.7 & $19(2)$ & & E2 \\
\hline & & 536.4 & $1.6(3)$ & & $M 1 / E 2$ \\
\hline \multirow[t]{2}{*}{$41 / 2^{+}$} & 3819.9 & 727.0 & $27(1)$ & & E2 \\
\hline & & 190.2 & $0.5(3)$ & & $M 1 / E_{2}$ \\
\hline \multirow[t]{2}{*}{$43 / 2^{+}$} & 4394.1 & 764.4 & $12(3)$ & & E2 \\
\hline & & 574.4 & $1.6(9)$ & & $M 1 / E_{2}$ \\
\hline $45 / 2^{+}$ & 4594.4 & 774.5 & $18(1)$ & & E2 \\
\hline $47 / 2^{+}$ & 5204.7 & 810.7 & $6.9(7)$ & & E2 \\
\hline $49 / 2^{+}$ & 5378.9 & 784.5 & $10(1)$ & & E2 \\
\hline $51 / 2^{+}$ & 6067.3 & 862.9 & $2.8(6)$ & & E2 \\
\hline $53 / 2^{+}$ & 6179.3 & 800.4 & $5.5(1)$ & & E2 \\
\hline $55 / 2^{+}$ & 6976.0 & 908.7 & $1.9(5)$ & & E2 \\
\hline $57 / 2^{+}$ & 7042.2 & 862.9 & $3.9(7)$ & & E2 \\
\hline $59 / 2^{+}$ & 7938.2 & 962.2 & $1.6(5)$ & $0.83(11)$ & E2 \\
\hline $61 / 2^{+}$ & 7972.6 & 930.4 & $2.8(9)$ & & E2 \\
\hline $63 / 2^{+}$ & 8944.4 & 1006.2 & $0.4(4)$ & & E2 \\
\hline $65 / 2^{+}$ & 8964.8 & 992.2 & $1.7(8)$ & $1.1(1)$ & E2 \\
\hline $67 / 2^{+}$ & 9892.4 & 948.0 & $0.1(3)$ & & E2 \\
\hline $69 / 2^{+}$ & 10010.9 & 1046.1 & $0.9(5)$ & $1.3(1)$ & E2 \\
\hline $71 / 2^{+}$ & 10857 & 964.6 & $1.9(6)$ & & E2 \\
\hline $73 / 2^{+}$ & 11099.0 & 1088.1 & $0.4(3)$ & & E2 \\
\hline $75 / 2^{+}$ & 11860.1 & 1002.3 & $0.1(5)$ & & E2 \\
\hline $77 / 2^{+}$ & 12198.9 & 1099.9 & $<0.3$ & & E2 \\
\hline $79 / 2^{+}$ & 12911.0 & 1051 & $<0.3$ & & E2 \\
\hline \multirow[t]{2}{*}{$81 / 2^{+}$} & 13331.9 & 1133 & $<0.3$ & & E2 \\
\hline & Bands $\mathbf{E} \& \mathbf{F}$ & & & & \\
\hline $1 / 2^{-}$ & $22.2^{c}$ & & & & \\
\hline $3 / 2^{-}$ & 88.6 & 66.4 & & & $M 1 / E_{2}$ \\
\hline $5 / 2^{-}$ & 102.7 & 80.5 & & & E2 \\
\hline \multirow[t]{2}{*}{$7 / 2^{-}$} & 254.4 & 165.8 & $6.9(6)$ & $0.80(13)$ & E2 \\
\hline & & 152.3 & $6.2(7)$ & $0.77(6)$ & $M 1 / E 2$ \\
\hline $9 / 2^{-}$ & 278.1 & 175.4 & $32(2)$ & $0.87(12)$ & E2 \\
\hline $11 / 2^{-}$ & 508.3 & 253.9 & $17(1)$ & $1.04(3)$ & E2 \\
\hline & & 230.4 & $2.5(5)$ & $0.96(6)$ & $M 1 / E_{2}$ \\
\hline $13 / 2^{-}$ & 536.9 & 258.8 & $49(1)$ & $1.14(10)$ & E2 \\
\hline $15 / 2^{-}$ & 838.4 & 330.1 & $22(2)$ & $1.1(1)$ & E2 \\
\hline & & 301.0 & $1.7(9)$ & $0.96(13)$ & $M 1 / E_{2}$ \\
\hline
\end{tabular}


TABLE I (Continued.)

\begin{tabular}{|c|c|c|c|c|c|}
\hline$J_{i}^{\pi}$ & $\mathrm{E}_{i}(\mathrm{keV})$ & $\mathrm{E}_{\gamma}(\mathrm{keV})^{a}$ & $I_{\gamma}{ }^{b}$ & $\mathrm{R}_{D C O}$ & Assignment \\
\hline $17 / 2^{-}$ & 867.3 & 330.4 & $74(1)$ & $1.09(7)$ & E2 \\
\hline \multirow[t]{2}{*}{$19 / 2^{-}$} & 1234.4 & 396.0 & $17(1)$ & $1.3(5)$ & E2 \\
\hline & & 367.8 & $1.0(7)$ & & $M 1 / E_{2}$ \\
\hline $21 / 2^{-}$ & 1257.3 & 390.0 & $75(1)$ & $1.16(11)$ & E2 \\
\hline \multirow[t]{2}{*}{$23 / 2^{-}$} & 1688.8 & 454.4 & $16(1)$ & $1.4(2)$ & E2 \\
\hline & & 432.0 & $0.8(7)$ & & $M 1 / E^{2}$ \\
\hline $25 / 2^{-}$ & 1697.4 & 440.1 & $73(1)$ & $1.23(8)$ & E2 \\
\hline $29 / 2^{-}$ & 2183.8 & 486.4 & $62(1)$ & $1.21(12)$ & E2 \\
\hline \multirow[t]{2}{*}{$27 / 2^{-}$} & 2196.1 & 507.3 & $17(1)$ & $1.25(10)$ & E2 \\
\hline & & 498.5 & $1.8(5)$ & $1.10(3)$ & $M 1 / E 2$ \\
\hline $33 / 2^{-}$ & 2712.0 & 528.2 & $54(1)$ & & E2 \\
\hline \multirow[t]{2}{*}{$31 / 2^{-}$} & 2753.4 & 557.3 & $16(2)$ & & E2 \\
\hline & & 568.9 & $0.9(1)$ & & $M 1 / E_{2}$ \\
\hline $37 / 2^{-}$ & 3283.4 & 571.4 & $\sim 50$ & & E2 \\
\hline \multirow[t]{2}{*}{$35 / 2^{-}$} & 3356.9 & 603.5 & $15(2)$ & & E2 \\
\hline & & 645.5 & $<0.3$ & & $M 1 / E 2$ \\
\hline $41 / 2^{-}$ & 3904.3 & 621.4 & $44(1)$ & & E2 \\
\hline \multirow[t]{2}{*}{$39 / 2^{-}$} & 3999.5 & 642.6 & $9.7(7)$ & & E2 \\
\hline & & 716.0 & $<0.3$ & & $M 1 / E_{2}$ \\
\hline $45 / 2^{-}$ & 4582.5 & 678.2 & $32(1)$ & & E2 \\
\hline \multirow[t]{2}{*}{$43 / 2^{-}$} & 4679.4 & 679.9 & $7.7(8)$ & & E2 \\
\hline & & 774.7 & $<0.3$ & & $M 1 / E_{2}$ \\
\hline $49 / 2^{-}$ & 5320.8 & 738.3 & $26(1)$ & & E2 \\
\hline \multirow{2}{*}{$47 / 2^{-}$} & 5414.2 & 734.8 & $5.1(5)$ & & E2 \\
\hline & & 831.1 & $<0.3$ & & $M 1 / E 2$ \\
\hline $53 / 2^{-}$ & 6120.4 & 799.6 & $12(1)$ & & E2 \\
\hline $51 / 2^{-}$ & 6209.8 & 795.6 & $2.5(6)$ & & E2 \\
\hline $57 / 2^{-}$ & 6980.9 & 860.5 & $10(1)$ & & E2 \\
\hline $55 / 2^{-}$ & 7071.4 & 861.6 & $1.8(6)$ & & E2 \\
\hline $61 / 2^{-}$ & 7902.2 & 921.3 & $4.7(5)$ & & E2 \\
\hline $59 / 2^{-}$ & 7988.2 & 916.8 & $0.8(3)$ & $1.15(3)$ & E2 \\
\hline $65 / 2^{-}$ & 8882.4 & 980.2 & $3.2(6)$ & & E2 \\
\hline $63 / 2^{-}$ & 8962.2 & 974.0 & $0.5(3)$ & $1.12(4)$ & E2 \\
\hline $69 / 2^{-}$ & 9916.9 & 1034.5 & $2.2(5)$ & & E2 \\
\hline $67 / 2^{-}$ & 9978 & 1016 & $0.3(3)$ & $1.04(5)$ & E2 \\
\hline $73 / 2^{-}$ & 10995.4 & 1078.5 & $1.2(6)$ & $0.9(3)$ & E2 \\
\hline $71 / 2^{-}$ & 11028 & 1050 & $<0.3$ & & E2 \\
\hline $77 / 2^{-}$ & 12104.3 & 1108.9 & $0.5(6)$ & $1.5(7)$ & E2 \\
\hline $81 / 2^{-}$ & 13240.0 & 1135.7 & $0.3(4)$ & & E2 \\
\hline $85 / 2^{-}$ & 14407 & 1167 & $<0.3$ & & E2 \\
\hline \multirow[t]{2}{*}{$89 / 2^{-}$} & 15602 & 1195 & $<0.3$ & & E2 \\
\hline & Bands $\mathbf{G} \& \mathbf{H}$ & & & & \\
\hline $5 / 2^{-}$ & $49.7^{c}$ & & & & $E 1^{c}$ \\
\hline $7 / 2^{-}$ & 141.9 & 92.2 & & & E2 \\
\hline \multirow[t]{2}{*}{$9 / 2^{-}$} & 258.8 & 209.1 & $1.8(6)$ & $0.7(2)$ & E2 \\
\hline & & 116.3 & $2.4(4)$ & $0.60(4)$ & $M 1 / E 2$ \\
\hline \multirow[t]{2}{*}{$11 / 2^{-}$} & 399.0 & 257.1 & $1.3(7)$ & $0.86(12)$ & E2 \\
\hline & & 139.6 & $0.8(5)$ & $0.52(10)$ & $M 1 / E 2$ \\
\hline \multirow[t]{2}{*}{$13 / 2^{-}$} & 561.2 & 302.4 & $2.0(8)$ & $0.99(3)$ & E2 \\
\hline & & 161.6 & $2.6(3)$ & $0.5(1)$ & $M 1 / E 2$ \\
\hline \multirow[t]{2}{*}{$15 / 2^{-}$} & 742.0 & 343.0 & $3.7(2)$ & $0.99(2)$ & E2 \\
\hline & & 180.4 & $1.2(6)$ & $0.53(8)$ & $M 1 / E_{2}$ \\
\hline \multirow[t]{2}{*}{$17 / 2^{-}$} & 940.8 & 379.6 & $2.3(2)$ & $1.04(4)$ & E2 \\
\hline & & 198.3 & $0.8(4)$ & $0.6(1)$ & $M 1 / E_{2}$ \\
\hline \multirow[t]{2}{*}{$19 / 2^{-}$} & 1153.8 & 411.8 & $4.6(7)$ & $1.06(5)$ & E2 \\
\hline & & 213.5 & $0.9(2)$ & & $M 1 / E_{2}$ \\
\hline \multirow[t]{3}{*}{$21 / 2^{-}$} & 1379.6 & 438.8 & $2.4(9)$ & $1.03(4)$ & E2 \\
\hline & & 225.4 & $0.4(5)$ & $0.70(2)$ & $M 1 / E 2$ \\
\hline & & 663.5 & $0.5(6)$ & & E1 \\
\hline $23 / 2^{-}$ & 1615.7 & 461.9 & $4.5(2)$ & $1.08(7)$ & E2 \\
\hline
\end{tabular}


TABLE I (Continued.)

\begin{tabular}{|c|c|c|c|c|c|}
\hline$J_{i}^{\pi}$ & $\mathrm{E}_{i}(\mathrm{keV})$ & $\mathrm{E}_{\gamma}(\mathrm{keV})^{a}$ & $I_{\gamma}^{b}$ & $\mathrm{R}_{D C O}$ & Assignment \\
\hline & & 235.8 & $0.8(2)$ & $0.9(1)$ & $M 1 / E 2$ \\
\hline & & 749.4 & $1.2(2)$ & $0.63(6)$ & $E 1$ \\
\hline \multirow[t]{3}{*}{$25 / 2^{-}$} & 1858.2 & 478.6 & $1.9(5)$ & 1.1(1) & E2 \\
\hline & & 241.9 & $0.2(1)$ & $0.53(11)$ & $M 1 / E 2$ \\
\hline & & 712.2 & $1.7(3)$ & $0.47(6)$ & E1 \\
\hline \multirow[t]{3}{*}{$27 / 2^{-}$} & 2113.4 & 497.7 & $6.0(5)$ & $1.17(5)$ & E2 \\
\hline & & 254.8 & $1.0(2)$ & $0.8(1)$ & $M 1 / E 2$ \\
\hline & & 806.7 & $1.1(2)$ & $0.69(9)$ & E1 \\
\hline \multirow[t]{3}{*}{$29 / 2^{-}$} & 2365.6 & 507.4 & $3.1(5)$ & & E2 \\
\hline & & 252.3 & $<0.3$ & & $M 1 / E 2$ \\
\hline & & 703.6 & $0.6(1)$ & $0.56(7)$ & E1 \\
\hline \multirow[t]{3}{*}{$31 / 2^{-}$} & 2641.5 & 528.1 & $3.0(5)$ & & E2 \\
\hline & & 276.0 & $0.4(2)$ & $0.7(8)$ & $M 1 / E 2$ \\
\hline & & 813.1 & $0.9(2)$ & $0.5(1)$ & E1 \\
\hline \multirow{3}{*}{$33 / 2^{-}$} & 2908.3 & 542.7 & $1.2(5)$ & & E2 \\
\hline & & 266.4 & $0.4(4)$ & $0.7(1)$ & $M 1 / E 2$ \\
\hline & & 653.2 & $0.7(3)$ & & E1 \\
\hline \multirow{3}{*}{$35 / 2^{-}$} & 3200.2 & 558.7 & $2.1(5)$ & & E2 \\
\hline & & 291.4 & $<0.3$ & $0.6(1)$ & $M 1 / E 2$ \\
\hline & & 774.0 & $0.7(6)$ & & $E 1$ \\
\hline \multirow[t]{3}{*}{$37 / 2^{-}$} & 3503.1 & 594.8 & $3.4(6)$ & & E2 \\
\hline & & 302.2 & $<0.3$ & & $M 1 / E 2$ \\
\hline & & 589.1 & $<0.3$ & & E1 \\
\hline \multirow[t]{3}{*}{$39 / 2^{-}$} & 3800.3 & 600.1 & $3.0(5)$ & & E2 \\
\hline & & 296.7 & $<0.3$ & & $M 1 / E 2$ \\
\hline & & 707.2 & $0.5(4)$ & & $E 1$ \\
\hline \multirow[t]{2}{*}{$41 / 2^{-}$} & 4155.0 & 651.9 & $0.8(5)$ & & E2 \\
\hline & & 354.2 & $<0.3$ & & $M 1 / E 2$ \\
\hline \multirow[t]{2}{*}{$43 / 2^{-}$} & 4456.2 & 655.9 & $1.1(5)$ & & E2 \\
\hline & & 636.2 & $<0.3$ & & E1 \\
\hline $45 / 2^{-}$ & 4862.1 & 707.1 & $0.6(4)$ & & E2 \\
\hline $47 / 2^{-}$ & 5175.8 & 719.6 & $1.6(3)$ & & E2 \\
\hline $49 / 2^{-}$ & 5626.3 & 764.2 & $0.3(2)$ & & E2 \\
\hline $51 / 2^{-}$ & 6961.7 & 785.9 & $1.2(2)$ & & E2 \\
\hline $53 / 2^{-}$ & 6449.4 & 823.1 & $0.3(3)$ & & E2 \\
\hline $55 / 2^{-}$ & 6812.7 & 851.0 & $0.9(3)$ & & E2 \\
\hline $57 / 2^{-}$ & 7327.8 & 878.4 & $<0.3$ & & E2 \\
\hline $59 / 2^{-}$ & 7724.9 & 912.2 & $0.9(2)$ & & E2 \\
\hline $61 / 2^{-}$ & 8274.2 & 946.4 & $<0.3$ & & E2 \\
\hline $63 / 2^{-}$ & 8698.8 & 973.9 & $0.4(3)$ & & E2 \\
\hline $65 / 2^{-}$ & 9266.5 & 992.3 & $<0.3$ & & E2 \\
\hline $67 / 2^{-}$ & 9714.9 & 1016.1 & $<0.3$ & & E2 \\
\hline $69 / 2^{-}$ & 10300 & 1033 & $<0.3$ & & E2 \\
\hline $71 / 2^{-}$ & 10761.9 & 1047.0 & $<0.3$ & & E2 \\
\hline $75 / 2^{-}$ & 11810.1 & 1048.2 & $<0.3$ & & E2 \\
\hline $79 / 2^{-}$ & 12890.1 & 1080.0 & $<0.3$ & & E2 \\
\hline $83 / 2^{-}$ & 14016.8 & 1126.7 & $<0.3$ & & E2 \\
\hline $87 / 2^{-}$ & 15196 & 1179 & $<0.3$ & & E2 \\
\hline $91 / 2^{-}$ & 16432 & 1236 & $<0.3$ & & E2 \\
\hline \multirow[t]{2}{*}{$\left(95 / 2^{-}\right)$} & 17733 & 1301 & $<0.3$ & & (EQ) \\
\hline & Band HK1 & & & & \\
\hline \multirow[t]{4}{*}{$19 / 2^{+}$} & 1644.6 & 778.1 & $1.1(6)$ & & M1 \\
\hline & & 928.2 & $2.5(8)$ & & M1 \\
\hline & & 1132.4 & $10(1)$ & & M1 \\
\hline & & 1262.3 & $0.9(6)$ & & E2 \\
\hline $21 / 2^{+}$ & 1793.9 & 149.3 & $6.6(6)$ & $0.92(6)$ & $M 1 / E 2$ \\
\hline \multirow[t]{2}{*}{$23 / 2^{+}$} & 1977.0 & 332.4 & $1.2(4)$ & $1.5(6)$ & E2 \\
\hline & & 183.1 & $8.8(6)$ & $0.96(5)$ & $M 1 / E 2$ \\
\hline \multirow[t]{2}{*}{$25 / 2^{+}$} & 2188.6 & 394.7 & $2.2(6)$ & & E2 \\
\hline & & 211.6 & $6.6(5)$ & $0.94(5)$ & $M 1 / E 2$ \\
\hline
\end{tabular}


TABLE I (Continued.)

\begin{tabular}{|c|c|c|c|c|c|}
\hline$J_{i}^{\pi}$ & $\mathrm{E}_{i}(\mathrm{keV})$ & $\overline{\mathrm{E}_{\gamma}(\mathrm{keV})^{a}}$ & $I_{\gamma}^{b}$ & $\mathrm{R}_{D C O}$ & Assignment \\
\hline \multirow[t]{2}{*}{$27 / 2^{+}$} & 2425.4 & 448.4 & $5.8(6)$ & & E2 \\
\hline & & 237.1 & $12(1)$ & & $M 1 / E^{2}$ \\
\hline \multirow[t]{2}{*}{$29 / 2^{+}$} & 2685.0 & 496.4 & $5.2(3)$ & & E2 \\
\hline & & 259.4 & $10(1)$ & $1.1(1)$ & $M 1 / E^{2}$ \\
\hline \multirow[t]{2}{*}{$31 / 2^{+}$} & 2966.1 & 540.7 & $8.5(9)$ & & E2 \\
\hline & & 281.0 & $9.6(8)$ & & $M 1 / E^{2}$ \\
\hline \multirow[t]{2}{*}{$33 / 2^{+}$} & 3265.6 & 580.6 & $8.8(7)$ & & E2 \\
\hline & & 299.6 & $2.2(6)$ & & $M 1 / E^{2}$ \\
\hline \multirow[t]{2}{*}{$35 / 2^{+}$} & 3584.0 & 617.9 & $6.6(7)$ & & E2 \\
\hline & & 318.4 & $2.3(6)$ & & $M 1 / E_{2}$ \\
\hline \multirow{2}{*}{$37 / 2^{+}$} & 3919.8 & 654.2 & $9.1(9)$ & & E2 \\
\hline & & 335.8 & & & $M 1 / E_{2}$ \\
\hline \multirow[t]{2}{*}{$39 / 2^{+}$} & 4262.0 & 678.0 & $6.3(6)$ & & E2 \\
\hline & & 342.1 & $2.8(7)$ & & $M 1 / E_{2}$ \\
\hline \multirow[t]{2}{*}{$41 / 2^{+}$} & 4614.9 & 695.1 & $6.4(9)$ & & E2 \\
\hline & & 352.9 & $\sim 2$ & & $M 1 / E_{2}$ \\
\hline \multirow[t]{2}{*}{$43 / 2^{+}$} & 4965.0 & 703.0 & $6.4(8)$ & & E2 \\
\hline & & 350.1 & $2.4(6)$ & & $M 1 / E_{2}$ \\
\hline \multirow[t]{2}{*}{$45 / 2^{+}$} & 5302.1 & 687.2 & $\sim 3$ & & E2 \\
\hline & & 337.1 & & & $M 1 / E 2$ \\
\hline \multirow{2}{*}{$47 / 2^{+}$} & 5639.7 & 674.7 & $9.6(2)$ & & E2 \\
\hline & & 337.8 & & & $M 1 / E 2$ \\
\hline \multirow[t]{2}{*}{$49 / 2^{+}$} & 5989.1 & 687.0 & $\sim 1$ & & E2 \\
\hline & & 349.4 & $\sim 0.7$ & & $M 1 / E_{2}$ \\
\hline \multirow[t]{2}{*}{$51 / 2^{+}$} & 6354.6 & 714.9 & $2.1(4)$ & & E2 \\
\hline & & 365.5 & $1.3(7)$ & & $M 1 / E_{2}$ \\
\hline \multirow[t]{2}{*}{$53 / 2^{+}$} & 6735.3 & 746.2 & $0.5(8)$ & & E2 \\
\hline & & 380.7 & $0.3(6)$ & & $M 1 / E_{2}$ \\
\hline \multirow[t]{2}{*}{$55 / 2^{+}$} & 7134.5 & 779.9 & $<0.3$ & & E2 \\
\hline & & 399.2 & $<0.3$ & & $M 1 / E 2$ \\
\hline \multirow[t]{2}{*}{$57 / 2^{+}$} & 7550.8 & 815.5 & $<0.3$ & & E2 \\
\hline & & 416.3 & $<0.3$ & & $M 1 / E_{2}$ \\
\hline $59 / 2^{+}$ & 7585.9 & 851.4 & $<0.3$ & & E2 \\
\hline & & 435.1 & $<0.3$ & & $M 1 / E 2$ \\
\hline $61 / 2^{+}$ & 8436.7 & 885.9 & $<0.3$ & & E2 \\
\hline & & 450.8 & $<0.3$ & & $M 1 / E^{2}$ \\
\hline $63 / 2^{+}$ & 8905 & 919 & $<0.3$ & & E2 \\
\hline & & 468 & $<0.3$ & & $M 1 / E_{2}$ \\
\hline $65 / 2^{+}$ & 9386 & 950 & $<0.3$ & & E2 \\
\hline & & 482 & $<0.3$ & & $M 1 / E_{2}$ \\
\hline & Band HK2 & & & & \\
\hline $23 / 2^{-}$ & 1984.2 & 190.3 & $2.1(4)$ & & $M 1 / E 2$ \\
\hline $25 / 2^{-}$ & 2161.6 & 177.4 & $6.3(5)$ & & $M / E^{2}$ \\
\hline $27 / 2^{-}$ & 2371.8 & 387.6 & $1.5(4)$ & & E2 \\
\hline & & 210.2 & $7.0(7)$ & & $M 1 / E_{2}$ \\
\hline $29 / 2^{-}$ & 2611.0 & 449.4 & $0.9(6)$ & & E2 \\
\hline & & 239.3 & $4.5(6)$ & & $M 1 / E_{2}$ \\
\hline $31 / 2^{-}$ & 2876.4 & 504.6 & $3.1(7)$ & & E2 \\
\hline & & 265.3 & $7.1(9)$ & & $M 1 / E^{2}$ \\
\hline $33 / 2^{-}$ & 3165.8 & 554.8 & $2.4(5)$ & & E2 \\
\hline & & 289.4 & $5.1(8)$ & & $M 1 / E 2$ \\
\hline $35 / 2^{-}$ & 3476.7 & 600.3 & $1.7(7)$ & & E2 \\
\hline & & 310.9 & $3.4(3)$ & & $M 1 / E^{2}$ \\
\hline $37 / 2^{-}$ & 3807.9 & 642.1 & $1.7(8)$ & & E2 \\
\hline & & 331.2 & $3.5(7)$ & & $M 1 / E_{2}$ \\
\hline $39 / 2^{-}$ & 4156.7 & 680.0 & $1.4(6)$ & & E2 \\
\hline & & 348.8 & $2.1(6)$ & & $M 1 / E_{2}$ \\
\hline $41 / 2^{-}$ & 4523.3 & 715.4 & $2.1(6)$ & & E2 \\
\hline & & 366.6 & $1.4(6)$ & & $M 1 / E_{2}$ \\
\hline $43 / 2^{-}$ & 4903.7 & 747.0 & $0.9(4)$ & & E2 \\
\hline
\end{tabular}


TABLE I (Continued.)

\begin{tabular}{|c|c|c|c|c|c|}
\hline$J_{i}^{\pi}$ & $\overline{\mathrm{E}_{i}(\mathrm{keV})}$ & $\mathrm{E}_{\gamma}(\mathrm{keV})^{a}$ & $I_{\gamma}^{b}$ & $\mathrm{R}_{D C O}$ & Assignment \\
\hline & & 380.4 & $1.1(7)$ & & $M 1 / E 2$ \\
\hline \multirow{2}{*}{$45 / 2^{-}$} & 5295.9 & 772.6 & $1.6(7)$ & & E2 \\
\hline & & 392.2 & $2.1(6)$ & & $M 1 / E 2$ \\
\hline \multirow[t]{2}{*}{$47 / 2^{-}$} & 5696.1 & 792.4 & $0.9(7)$ & & E2 \\
\hline & & 400.3 & & & $M 1 / E 2$ \\
\hline \multirow[t]{2}{*}{$49 / 2^{-}$} & 6095.8 & 799.9 & $0.8(6)$ & & E2 \\
\hline & & 399.6 & & & $M 1 / E 2$ \\
\hline \multirow[t]{2}{*}{$51 / 2^{-}$} & 6481.0 & 784.9 & $0.6(6)$ & & E2 \\
\hline & & 385.3 & $<0.3$ & & $M 1 / E 2$ \\
\hline \multirow[t]{2}{*}{$53 / 2^{-}$} & 6862 & 766 & $0.5(7)$ & & E2 \\
\hline & & 381 & $<0.3$ & & $M 1 / E 2$ \\
\hline \multirow[t]{2}{*}{$55 / 2^{-}$} & 7235.4 & 754.4 & $0.4(3)$ & & E2 \\
\hline & & 373 & $<0.3$ & & M1/E2 \\
\hline \multirow[t]{2}{*}{$59 / 2^{-}$} & 8055 & 820 & $0.3(6)$ & & E2 \\
\hline & Band X1 & & & & \\
\hline \multirow[t]{2}{*}{$33 / 2^{-}$} & 3090.4 & 906.6 & $<0.3$ & & E2 \\
\hline & & 378.4 & $0.6(4)$ & & M1 \\
\hline \multirow[t]{3}{*}{$37 / 2^{-}$} & 3642.4 & 552.0 & $1.2(5)$ & $1.1(2)$ & E2 \\
\hline & & 930.4 & $0.4(3)$ & $1.0(1)$ & E2 \\
\hline & & 359.1 & $0.6(5)$ & & M1 \\
\hline \multirow{3}{*}{$41 / 2^{-}$} & 4261.4 & 619.0 & $1.3(5)$ & & E2 \\
\hline & & 978.1 & $<0.3$ & $0.92(9)$ & E2 \\
\hline & & 356.7 & $0.4(3)$ & $0.92(5)$ & M1 \\
\hline \multirow[t]{3}{*}{$45 / 2^{-}$} & 4944.6 & 683.2 & $3.6(7)$ & $0.94(2)$ & E2 \\
\hline & & 1039.9 & $0.4(3)$ & $0.98(5)$ & E2 \\
\hline & & 362.0 & $0.6(3)$ & $0.99(5)^{d}$ & M1 \\
\hline \multirow[t]{3}{*}{$49 / 2^{-}$} & 5682.9 & 738.3 & $4.4(8)$ & $0.97(2)$ & E2 \\
\hline & & 1100.0 & 0.3 & & E2 \\
\hline & & 361.7 & $0.6(3)$ & $0.99(5)^{d}$ & M1 \\
\hline \multirow[t]{3}{*}{$53 / 2^{-}$} & 6469.6 & 786.7 & $4.4(8)$ & $0.88(5)$ & E2 \\
\hline & & 1148.4 & $<0.3$ & & E2 \\
\hline & & 348.8 & $0.3(1)$ & & M1 \\
\hline \multirow[t]{2}{*}{$57 / 2^{-}$} & 7300.8 & 831.2 & $4.2(6)$ & $1.11(5)$ & E2 \\
\hline & & 1180.0 & $<0.3$ & & E2 \\
\hline $61 / 2^{-}$ & 8171.2 & 870.4 & $2.5(5)$ & $0.99(4)$ & E2 \\
\hline $65 / 2^{-}$ & 9092.5 & 921.3 & $1.8(3)$ & $1.06(3)$ & E2 \\
\hline $69 / 2^{-}$ & 10073.9 & 981.4 & $1.4(3)$ & $1.02(5)$ & E2 \\
\hline $73 / 2^{-}$ & 11112.1 & 1038.2 & $0.9(4)$ & $1.01(7)$ & E2 \\
\hline $77 / 2^{-}$ & 12200 & 1088 & $0.5(2)$ & & E2 \\
\hline \multirow[t]{2}{*}{$81 / 2^{-}$} & 13314 & 1114 & $<0.3$ & & E2 \\
\hline & Band X2 & & & & \\
\hline $33 / 2^{+}$ & 2882.9 & 456.7 & $<0.3$ & & \\
\hline \multirow[t]{4}{*}{$37 / 2^{+}$} & 3481.3 & 598.4 & $<0.3$ & $1.0(1)$ & E2 \\
\hline & & 1055.1 & $2.0(2)$ & $0.99(6)$ & E2 \\
\hline & & 567.4 & $0.3(1)$ & $0.74(8)$ & M1 \\
\hline & & 388.2 & $1.7(1)$ & $1.11(6)$ & M1 \\
\hline $41 / 2^{+}$ & 4087.5 & 607.2 & $0.3(3)$ & $1.0(1)$ & E2 \\
\hline & & 994.8 & $2.0(2)$ & $0.98(5)$ & E2 \\
\hline & & 458.1 & $<0.3$ & $0.46(6)$ & M1 \\
\hline & & 267.8 & $<0.3$ & & \\
\hline $45 / 2^{+}$ & 4736.5 & 649.0 & $0.4(4)$ & $0.99(3)$ & E2 \\
\hline & & 916.5 & $3.0(4)$ & $1.01(5)$ & E2 \\
\hline $49 / 2^{+}$ & 5493.3 & 756.8 & $1.2(4)$ & $1.03(4)$ & E2 \\
\hline & & 898.8 & $4.5(3)$ & $1.1(1)$ & E2 \\
\hline & Band X3 & & & & \\
\hline $53 / 2^{+}$ & 6340.0 & 846.7 & $0.7(2)$ & $0.86(4)$ & E2 \\
\hline $57 / 2^{+}$ & 7183.9 & 843.9 & $0.3(1)$ & $0.89(4)$ & E2 \\
\hline $61 / 2^{+}$ & 8080.1 & 896.2 & $0.3(1)$ & $1.0(1)$ & E2 \\
\hline $65 / 2^{+}$ & 9042.1 & 962.0 & $<0.3$ & $0.95(5)$ & E2 \\
\hline $69 / 2^{+}$ & 10060 & 1018 & $<0.3$ & $0.89(5)$ & E2 \\
\hline
\end{tabular}


TABLE I (Continued.)

\begin{tabular}{|c|c|c|c|c|c|}
\hline$J_{i}^{\pi}$ & $\overline{\mathrm{E}_{i}(\mathrm{keV})}$ & $\overline{\mathrm{E}_{\gamma}(\mathrm{keV})^{a}}$ & $I_{\gamma}{ }^{b}$ & $\mathrm{R}_{D C O}$ & Assignment \\
\hline $73 / 2^{+}$ & 11112 & 1052 & $<0.3$ & $1.1(1)$ & E2 \\
\hline \multirow[t]{2}{*}{$77 / 2^{+}$} & 12187 & 1075 & $<0.3$ & & E2 \\
\hline & Band X4 & & & & \\
\hline $53 / 2^{+}$ & 6401.1 & 907.8 & $0.7(3)$ & $0.99(4)^{e}$ & E2 \\
\hline \multirow[t]{2}{*}{$57 / 2^{+}$} & 7310.0 & 908.9 & $0.6(3)$ & $0.99(4)^{e}$ & E2 \\
\hline & & 970.0 & $0.6(4)$ & & E2 \\
\hline $61 / 2^{+}$ & 8281 & 971 & $0.3(3)$ & $0.90(6)$ & E2 \\
\hline $65 / 2^{+}$ & 9277 & 996 & $0.3(3)$ & $1.1(1)$ & E2 \\
\hline \multirow[t]{2}{*}{$69 / 2^{+}$} & 10229 & 952 & $<0.3$ & & E2 \\
\hline & Band MAB & & & & \\
\hline$(37 / 2)$ & 3428.8 & 515.0 & $<0.3$ & & \\
\hline \multirow[t]{2}{*}{$37 / 2^{-}$} & 4069.9 & 554.4 & $<0.3$ & & \\
\hline & & 641.1 & $0.6(2)$ & & (E2) \\
\hline \multirow{2}{*}{$41 / 2^{-}$} & 4678.1 & 608.2 & $1.1(3)$ & $0.89(3)$ & E2 \\
\hline & & 1048.4 & $<0.3$ & & (E1) \\
\hline $45 / 2^{-}$ & 5330.4 & 652.3 & $0.6(3)$ & $1.10(5)$ & E2 \\
\hline \multirow[t]{2}{*}{$49 / 2^{-}$} & 6042.3 & 711.9 & $0.4(3)$ & $0.99(4)$ & E2 \\
\hline & & 837.6 & $1.5(3)$ & $0.66(6)$ & E1 \\
\hline $53 / 2^{-}$ & 6828.9 & 786.6 & $1.2(3)$ & $0.90(5)$ & E2 \\
\hline $57 / 2^{-}$ & 7684.7 & 855.8 & $0.8(3)$ & $1.03(4)$ & E2 \\
\hline $61 / 2^{-}$ & 8610.9 & 926.2 & $0.4(2)$ & $0.91(6)$ & E2 \\
\hline $65 / 2^{-}$ & 9601.0 & 990.1 & $0.3(2)$ & $0.95(6)$ & E2 \\
\hline $69 / 2^{-}$ & 10645.5 & 1044.5 & $<0.3$ & & E2 \\
\hline $73 / 2^{-}$ & 11729.1 & 1083.6 & $<0.3$ & & E2 \\
\hline \multirow[t]{2}{*}{$77 / 2^{-}$} & 12829 & 1100 & $<0.3$ & & E2 \\
\hline & Band ED1 & & & & \\
\hline$(39 / 2)^{+}$ & 4571.1 & 1287.7 & $<0.3$ & & (E1) \\
\hline \multirow[t]{2}{*}{$43 / 2^{+}$} & 5139.5 & 568.4 & $0.7(1)$ & & (E2) \\
\hline & & 1234.7 & $0.5(2)$ & $0.53(2)$ & E1 \\
\hline \multirow[t]{2}{*}{$47 / 2^{+}$} & 5699.9 & 560.4 & $1.9(2)$ & $0.90(1)$ & E2 \\
\hline & & 1117.4 & $0.9(1)$ & $0.51(2)$ & E1 \\
\hline $51 / 2^{+}$ & 6327.3 & 627.4 & $6.1(3)$ & $1.08(8)$ & E2 \\
\hline $55 / 2^{+}$ & 7006.9 & 679.6 & $8.1(4)$ & $0.97(7)$ & E2 \\
\hline $59 / 2^{+}$ & 7743.7 & 736.8 & $7.0(4)$ & $0.99(9)$ & E2 \\
\hline $63 / 2^{+}$ & 8539.2 & 795.5 & $6.3(3)$ & $0.98(8)$ & E2 \\
\hline $67 / 2^{+}$ & 9394.9 & 855.7 & $5.3(4)$ & $0.9(1)$ & E2 \\
\hline $71 / 2^{+}$ & 10309.1 & 914.2 & $4.4(4)$ & $1.0(1)$ & E2 \\
\hline $75 / 2^{+}$ & 11280.7 & 971.6 & $3.5(6)$ & $0.90(9)$ & E2 \\
\hline $79 / 2^{+}$ & 12306.6 & 1025.9 & $2.8(5)$ & $0.95(5)$ & E2 \\
\hline $83 / 2^{+}$ & 13385.8 & 1079.2 & $1.8(4)$ & $1.04(8)$ & E2 \\
\hline $87 / 2^{+}$ & 14515.7 & 1129.9 & $2.0(2)$ & $0.92(9)$ & E2 \\
\hline $91 / 2^{+}$ & 15698.4 & 1182.7 & $1.2(3)$ & $1.1(1)$ & E2 \\
\hline $95 / 2^{+}$ & 16933.9 & 1235.5 & $0.5(2)$ & $0.9(1)$ & E2 \\
\hline $99 / 2^{+}$ & 18226.5 & 1292.6 & $0.5(2)$ & & E2 \\
\hline $103 / 2^{+}$ & 19575.2 & 1348.7 & $<0.3$ & & E2 \\
\hline \multirow[t]{2}{*}{$107 / 2^{+}$} & 20981 & 1406 & $<0.3$ & & E2 \\
\hline & Band ED2 & & & & \\
\hline$(\mathrm{X}+2)$ & $Y+717.5$ & 717.5 & $1.0(7)$ & & (E2) \\
\hline$(\mathrm{X}+4)$ & $Y+1490.0$ & 772.5 & $0.9(7)$ & & (E2) \\
\hline$(\mathrm{X}+6)$ & $Y+2321.7$ & 831.7 & $1.2(9)$ & & (E2) \\
\hline$(\mathrm{X}+8)$ & $Y+3213.8$ & 892.1 & $1.1(8)$ & & (E2) \\
\hline$(\mathrm{X}+10)$ & $Y+4167.6$ & 953.8 & $1.1(7)$ & & (E2) \\
\hline$(\mathrm{X}+12)$ & $Y+5184.3$ & 1016.7 & $0.9(9)$ & & (E2) \\
\hline$(\mathrm{X}+14)$ & $Y+6265.1$ & 1080.8 & $0.7(8)$ & & (E2) \\
\hline$(\mathrm{X}+16)$ & $Y+7409.8$ & 1144.7 & $0.5(6)$ & & (E2) \\
\hline$(\mathrm{X}+18)$ & $Y+8621.4$ & 1211.6 & $<0.3$ & & (E2) \\
\hline$(\mathrm{X}+20)$ & $Y+9899$ & 1278 & $<0.3$ & & (E2) \\
\hline
\end{tabular}


TABLE I (Continued.)

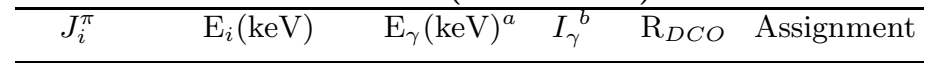

$[a]$ The uncertainties in the quoted $\gamma$-ray energies are $0.2 \mathrm{keV}$ for most transitions, and $0.5 \mathrm{keV}$ for weak transitions $\left(I_{\gamma}<1\right)$.

[b] Relative intensity of the transition with respect to the intensity of $522-\mathrm{keV}$ in band $\mathrm{A}$.

[c] Adopted values from the Table of Isotope [28].

[d] The DCO ratio was measured for the sum peak of $362.0-$ and $361.7-\mathrm{keV}$ transitions which depopulate the $45 / 2^{-}$and $49 / 2^{-}$levels.

[e] The DCO ratio was measured for the sum peak of 907.8- and 909.0-keV transitions in band X4.

\section{DISCUSSION}

Cranking calculations for ${ }^{171} \mathrm{Hf}$ have been carried out with the UC code [10-12]. Proton and neutron orbital energies for the ND region are displayed on the Routhian diagrams in Fig. 6. Table II lists the labeling of the quasiparticles and the related orbitals predicted by the UC code to be closest to the Fermi surface. Upper case letters represent quasineutrons and lower case letters quasiprotons. Each letter corresponds to a state described by a specific combination of asymptotic Nilsson orbitals with appropriate signature and parity quantum numbers. Configurations of the bands in ${ }^{171} \mathrm{Hf}$ are assigned below based on intrinsic properties of the bands, such as the observed alignments, crossings expected at specific frequencies, excitation energies, and on a systematic comparison of these configurations with structures observed in neighboring nuclei. In Fig. 7, the experimental alignments of all bands are displayed as a function of the rotational frequency. Harris parameters $J_{0}=35 \hbar^{2} / \mathrm{MeV}$ and $J_{1}=38 \hbar^{4} / \mathrm{MeV}^{3}$ were chosen to subtract the angular momentum of the core such that the ground-state band in ${ }^{170} \mathrm{Hf}$ [15] has nearly zero alignment at low frequencies, and nearly constant alignment following the first band crossing. The calculated and experimentally observed band crossings are summarized in Table III. Fig. 8 provides the excitation energies of all bands in ${ }^{171} \mathrm{Hf}$ relative to a rigid-rotor reference $A I(I+1)$, where the inertia parameter $A$ was chosen to be $7.5 \mathrm{keV}$.

TABLE II: Labels and alignments $\left(i_{x}\right)$ of theoretical Routhians for neutrons and protons. The spherical shell-model states represent only the main components of the wave functions if the orbitals are mixed.

\begin{tabular}{|c|c|c|c|c|c|}
\hline \multirow{2}{*}{$\begin{array}{c}\text { Spherical shell- } \\
\text { model state }\end{array}$} & \multirow{2}{*}{$\begin{array}{c}\text { Nilsson } \\
\text { orbital }\end{array}$} & \multicolumn{2}{|c|}{$\alpha=+1 / 2$} & \multicolumn{2}{|c|}{$\alpha=-1 / 2$} \\
\hline & & Label & $i_{x}$ & Label & $\overline{i_{x}}$ \\
\hline$\nu i_{13 / 2}$ & $\nu[633] \frac{7}{2}^{+}$ & $\bar{A}$ & 5.0 & B & 4.4 \\
\hline$\nu i_{13 / 2}$ & $\nu[642] \frac{5}{2}^{+}$ & $\mathrm{C}$ & 2.7 & $\mathrm{D}$ & 0.5 \\
\hline$\nu f_{5 / 2}$ & $\nu[521] \frac{1}{2}^{-}$ & $\mathrm{E}$ & 1.5 & $\mathrm{~F}$ & 0.3 \\
\hline$\nu f_{7 / 2}$ & $\nu[512] \frac{5}{2}^{-}$ & G & 0.6 & $\mathrm{H}$ & 0.6 \\
\hline$\nu h_{9 / 2}$ & $\nu[514] \frac{7}{2}^{-}$ & M & 0.2 & $\mathrm{~N}$ & 0.2 \\
\hline$\pi g_{7 / 2}$ & $\pi[404] \frac{7}{2}^{+}$ & $\mathrm{a}$ & 0.5 & b & 0.5 \\
\hline$\pi d_{5 / 2}$ & $\pi[402] \frac{5}{2}^{+}$ & c & 0 & $\mathrm{~d}$ & 0 \\
\hline$\pi i_{13 / 2}$ & $\pi[660] \frac{1}{2}+$ & $\mathrm{m}$ & 5.0 & & \\
\hline$\pi h_{11 / 2}$ & $\pi[514] \frac{9}{2}^{-}$ & e & 2.0 & $\mathrm{f}$ & 2.0 \\
\hline$\pi h_{9 / 2}$ & $\pi[541] \frac{1}{2}^{-}$ & $g$ & 3.6 & & \\
\hline
\end{tabular}

\section{A. Bands E, F, G, H, A, B and high-spin proton alignments}

The three strongest bands have been previously identified as being built on neutron [521]1/2- (E and F), [512]5/2$(\mathrm{G}$ and $\mathrm{H})$, and [633]7/2+ $(\mathrm{A}$ and $\mathrm{B})$ orbitals [21]. As seen in Fig.7, the negative-parity bands exhibit little initial aligned angular momenta which is consistent with the expectation for these orbitals. The $\mathrm{AB}$ crossings occur at a rotational frequency of $\hbar \omega \sim 250 \mathrm{keV}$ with a total gain of $\sim 9.5 \hbar$. The energy splitting between bands $\mathrm{E}$ and $\mathrm{F}$ is obvious, as can be seen in Fig. 8(a). The excitation energies of bands $\mathrm{G}$ and $\mathrm{H}$ lie between those of bands $\mathrm{E}$ and $\mathrm{F}$. For bands $\mathrm{A}$ and $\mathrm{B}$, the initial alignments are $\sim 2.5 \hbar$ and the $\mathrm{AB}$ crossing is blocked. The first crossing in band $\mathrm{A}$, at $\hbar \omega \sim 380 \mathrm{keV}$, with a gain of $\sim 6 \hbar$ is identified as the $\mathrm{BC}$ crossing. But the $\mathrm{BC}$ crossing is clearly missing in band $\mathrm{B}$ where, instead, a smooth gain of $\sim 5.7 \hbar$ in angular momentum is present in a frequency region between 330 and $500 \mathrm{keV}$. Theoretically, the first band crossing in band B should be associated with the AD neutron orbitals where a 
TABLE III: Summary of observed band crossings, crossing frequencies (in keV), and alignment gain $i_{x}$ (in $\hbar$ ) in ${ }^{171} \mathrm{Hf}$.

\begin{tabular}{|c|c|c|c|}
\hline \multicolumn{2}{|c|}{$\overline{\text { Band Crossing }}$} & $\overline{\hbar \omega}$ & $\overline{i_{x}}$ \\
\hline $\bar{A}$ & $\overline{\mathrm{BC}}$ & 380 & 6.0 \\
\hline \multirow[t]{2}{*}{ B } & $\mathrm{AD}$ & 380 & 5.7 \\
\hline & $\mathrm{fg}$ & 490 & $>6.4$ \\
\hline \multirow[t]{2}{*}{$\mathrm{E}$} & $\mathrm{AB}$ & 240 & 9.5 \\
\hline & fg & 510 & $>4.7$ \\
\hline $\mathrm{F}$ & $\mathrm{AB}$ & 250 & 9.5 \\
\hline G & $\mathrm{AB}$ & 250 & 9.5 \\
\hline \multirow[t]{2}{*}{$\mathrm{H}$} & $\mathrm{AB}$ & 250 & 9.5 \\
\hline & fg & 550 & 5.2 \\
\hline HK1 & $\mathrm{BC}$ & 320 & 6.0 \\
\hline $\mathrm{HK} 2$ & $\mathrm{BC}$ & 360 & 6.5 \\
\hline
\end{tabular}

strong interaction strength results in a gradual upbend. All these features are consistent with UC calculations in this study, and the previous total Routhian surface and Woods-Saxon cranked shell-model (CSM) calculations as well. For these three strongest bands, the configuration assignments agree with those proposed in the previous study of Ref. [21].

At a rotational frequency between 500 and $550 \mathrm{keV}$, another alignment is seen in every band that has been observed to sufficiently high spins, except in bands ED1 and ED2. The alignment gains deduced from Fig.7 for bands H and $\mathrm{B}$ are $5 \hbar$ and $6.8 \hbar$, respectively. It is larger than $5 \hbar$ in band $\mathrm{E}$, but the full alignment is not observed yet. This crossing must involve quasiprotons since it is observed as a general phenomenon present in bands associated with different neutron configurations. While the onset of a proton alignment at high spins has been observed in a number of nuclei in the mass region, the full alignments have only been delineated in a few neighboring nuclei such as ${ }^{166} \mathrm{Hf}$ [29], ${ }^{168} \mathrm{Hf}$ [25], and ${ }^{169} \mathrm{Hf}$ [30]. The proton alignments in these Hf nuclei are similar in their crossing frequencies and alignment gains, and have been identified as due to a mixed fg crossing (based on a mixture of the [514]9/2 $2^{-}$and [541]1/2- orbitals). The same may be concluded for ${ }^{171} \mathrm{Hf}$, as discussed below.

The lowest proton crossings predicted by the UC calculations are the ef (based on the $[514] 9 / 2^{-}$orbital) and the fg crossing that occurs in a similar frequency range. The large alignment gain observed in ${ }^{171} \mathrm{Hf}$ cannot be explained by the excitation of the e and $f$ quasiprotons alone. The $g$ quasiproton (based on the [541]1/2 $2^{-}$orbital), which has a large alignment and is predicted to cross the e orbital around $\hbar \omega \sim 430 \mathrm{keV}$ (see Fig. 6), must be involved as well. It should be noted that the calculated crossing frequency is very dependent on deformation, and the dispersion of the observed crossing frequencies can be attributed to this property. The $[541] 1 / 2^{-}$(g) orbital is close to the proton Fermi surface in ${ }_{72}^{171} \mathrm{Hf}$, and yrast bands in both ${ }_{71}^{167} \mathrm{Lu}[31]$ and ${ }_{73}^{171} \mathrm{Ta}[32]$ are based on this state, which is known to (i) drive nuclei to larger deformation and to (ii) cause a delay in the crossing frequency of the first neutron pair. The mixed fg crossing from the [514]9/2- and [541]1/2- orbitals is predicted to generate about $5.6 \hbar$ of aligned angular momentum, in general agreement with the observations. Therefore, at high spin, bands B, E, and $\mathrm{H}$ are assigned five-quasiparticle configurations involving the $\mathrm{f}$ and $\mathrm{g}$ quasiprotons. Such a mixed proton alignment has previously been suggested in other rare earth nuclei, such as ${ }^{165,166} \mathrm{Yb}$ [33], ${ }^{167,168} \mathrm{Yb}$ [34], and ${ }^{170} \mathrm{~W}$ [35]. Only in recent years, however, have the bands been observed to sufficiently high spins to delineate the full alignment in several nuclei.

Alternatively, one might consider the mb configuration, or $\pi[660] 1 / 2^{+}(\alpha=+1 / 2) \otimes \pi[404] 7 / 2^{+}(\alpha=-1 / 2)$. The UC calculations indicate that the alignment of this pair should occur around frequencies $\hbar \omega \sim 500 \mathrm{keV}$. The pronounced down-sloping of the $\pi[660] 1 / 2^{+}$orbital (see Fig. 6) as a function of rotational frequency is well known, and this state alone is expected to contribute about $5 \hbar$ to the alignment. This orbital has been identified in neighboring Lu isotopes $\left({ }^{163} \mathrm{Lu}[2,3],{ }^{165} \mathrm{Lu}[4]\right.$, and $\left.{ }^{167} \mathrm{Lu}[5]\right)$, where it drives nuclei to large deformations with significant triaxiality that can produce wobbling. The orbital has also been observed in neighboring Ta isotopes ${ }^{167} \mathrm{Ta}[7],{ }^{169} \mathrm{Ta}[36]$, and ${ }^{171} \mathrm{Ta}[32]$. In each of these cases, the $[660] 1 / 2^{+}$orbital is located higher than the $[541] 1 / 2^{-}$one, allowing the latter to align more easily. In ${ }^{171} \mathrm{Hf}$, the $\pi[660] 1 / 2^{+}$orbital has been identified in band ED1 [16]. As discussed below, this orbital drives the nucleus to an enhanced deformation relative to the ND structures. Therefore, the configuration mb can be ruled out, and the mixed fg crossing can be firmly assigned to the proton alignment at $\hbar \omega \sim 500 \mathrm{keV}$. This makes ${ }^{171} \mathrm{Hf}$ the second case, after ${ }^{168} \mathrm{Hf}[25]$, where both fg proton alignment and ED bands involving an aligned $\pi[660] 1 / 2^{+}$proton, have been established. 


\section{B. Band X1}

The lower part of band $\mathrm{X} 1$ has an alignment very similar to that of band $\mathrm{E}$ at rotational frequencies above the $\mathrm{AB}$ crossing, see Fig. 7(a). However, its excitation energy is $\sim 400 \mathrm{keV}$ above the EAB configuration, see Fig. 8(a). In addition, band X1 is observed to be strongly linked to band $\mathrm{E}$ by many transitions. These similarities and connections between the two bands indicate that the lower part of band X1 may correspond to a vibrational band based on the EAB configuration.

Both $\beta$ - and $\gamma$-vibrational bands have been observed in neighboring ${ }^{168,170,172} \mathrm{Hf}$ nuclei at low spins and they are connected to their respective ground-state bands [28]. These are quadrupole vibrations with $K=0$ and $K=2$, respectively. At higher spins, a $\beta$-vibrational band has been proposed in ${ }^{172} \mathrm{Hf}$ decaying to levels above the first neutron alignment in the ground-state band [37]. In ${ }^{169} \mathrm{Hf}$, however, $\gamma$-vibrational coupling has been reported [30] which is known to enhance the strength of the $E 2$ transitions to the band on which the $\gamma$-vibration is built. The decay-out is linked to a $\gamma$-vibrational component of the wave function, achieved by a $K=2$ coupling in the band's configuration. A similar picture of increased branching ratios has been discovered in ${ }^{163} \mathrm{Er}$ [38]. To find the $B(E 2)$ values for the decay-out transitions of band $\mathrm{X} 1$ in ${ }^{171} \mathrm{Hf}$, a transition quadrupole moment of $6.2 e \mathrm{~b}$ is assumed for the band, which is the same as the measured value for band $\mathrm{E}$ [21]. The average branching ratio between the decay-out stretched $E 2$ transitions and the in-band transitions is about $10 \%$, corresponding to $B(E 2)$ values for the decay-out transitions of $0.12 e^{2} b^{2}$, that is of the order of $20 \mathrm{~W}$. u. This is substantially higher than expected for $E 2$ decays, and is in the range of values expected for vibrational transitions, suggesting the possibility of a mixing with a $\gamma$-vibrational state. A possibility is that the band X1 may involve the quasi-neutron orbitals FG (and AB), where the configuration $\left\{[521] 1 / 2^{-} \otimes[512] 5 / 2^{-}\right\}_{K=2}$ couples with the $\mathrm{E}$ orbital, resulting in the correct parity. The vibrational excitation energy, $357 \mathrm{keV}$ between the two $41 / 2^{-}$states in bands $\mathrm{X} 1$ and $\mathrm{E}(\mathrm{AB})$, is substantially lower than the $588 \mathrm{keV}$ between the two $41 / 2^{+}$states in bands AMF and A(BC) of ${ }^{169} \mathrm{Hf}$ [30].

At $\hbar \omega \sim 410 \mathrm{keV}$, band X1 exhibits another alignment with a gain of $2.5 \hbar$ above that of the EAB configuration. This must be caused by an additional neutron alignment. The most probable candidate would be the CD crossing. According to UC calculations, this $\mathrm{CD}$ crossing occurs at a frequency higher than that of the $\mathrm{AB}$ and $\mathrm{BC}$ ones with a gain of $\sim 3 \hbar$ in angular momentum. Thus, band $\mathrm{X} 1$ is crossed by a five-quasineutron $\mathrm{E}(\mathrm{ABCD})$ configuration. However, why the CD crossing has not been observed systematically in other bands is not understood.

\section{Bands X2 - X4}

The positive-parity band X2 feeds bands A and B. Its structure is most likely based on the C orbital. The $\sim 9 \hbar$ alignment gain of the band at $\hbar \omega \sim 300 \mathrm{keV}$ is then due to the $\mathrm{AB}$ crossing, resulting in a $\mathrm{CAB}$ configuration above the initial alignment, and duplicates the $\mathrm{ABC}$ configuration in band $\mathrm{A}$ above $\hbar \omega \sim 380 \mathrm{keV}$. Therefore, band $\mathrm{X} 2$ is the low-spin continuation of the ABC configuration, as is demonstrated in Fig. 7(c). The exchange in character between bands X2 and A can also be seen in Fig. 8(b). Similar structure exchanges have been observed in ${ }^{167} \mathrm{Hf}[39]$.

Band X3 starts from a rotational frequency of $\hbar \omega \sim 420 \mathrm{keV}$ with an initial alignment $\sim 1 \hbar$ higher than that of the ABC configuration. The alignments are due to neutrons because the proton alignment starts at $\hbar \omega>500 \mathrm{keV}$. Furthermore, the configuration of band X3 most likely involves the positive-parity neutron orbitals A, B, and C so that it starts from such a high initial alignment. Therefore, the additional $\sim 1 \hbar$ alignment probably comes from the alignment of negative-parity orbitals, e.g., from the GH crossing. The $\mathrm{E}$ and $\mathrm{F}$ orbitals are predicted to align at a much higher frequency.

Band X4 is a short sequence with a positive parity. It feeds band X2 and then decays to band A. Fig. 8(b) indicates that band $\mathrm{X} 4$ is the continuation of the one-quasiparticle band $\mathrm{A}$, with little deviation. While sharp band crossings, such as $\mathrm{AB}$ and $\mathrm{BC}$, cause an obvious decrease in the slope of excitation energy curves with spin, a gradual alignment such as the $\mathrm{AD}$ crossing in band $\mathrm{B}$ only produces a very gentle deviation in its excitation energy curve. Therefore, band X4 may represent the continuation of band A, proceeding directly to the proton crossing at $\hbar \omega \sim 500 \mathrm{keV}$. Nevertheless, it should be noted that the possibility of a neutron CD alignment before the proton crossing cannot be ruled out.

\section{Band MAB}

The alignment pattern of band MAB is very similar to that of bands $\mathrm{E}, \mathrm{F}, \mathrm{G}$, and $\mathrm{H}$, indicating that this sequence may be associated with a three-quasiparticle configuration consisting of a single quasineutron orbital going through the $\mathrm{AB}$ crossing. The single quasineutron cannot be associated with the positive-parity orbitals $\mathrm{C}$ or $\mathrm{D}$, which would duplicate the $\mathrm{ABC}$ and $\mathrm{BAD}$ configurations in bands $\mathrm{A}$ and $\mathrm{B}$, respectively. Fig. 8(a) indicates that the excitation 
energy of band MAB is $\sim 700 \mathrm{keV}$ higher than that of band E around a spin of $\sim 30 \hbar$ or a rotational frequency of $\hbar \omega \sim 500 \mathrm{keV}$. In the quasiparticle Routhian diagram of Fig. 6 orbital M is $\sim 800 \mathrm{keV}$ higher than orbital E, roughly reproducing the energy difference between the MAB and EAB configurations. Thus, band MAB is most likely based on orbital M, the [514]7/2- Nilsson orbital, going through the AB crossing. The proposed configuration has a negative parity that is consistent with the parity assignment in section III-B based on experimentally measured quantities. The [514]7/2- orbital has been identified in excited structures of ${ }^{172} \mathrm{Hf}[37],{ }^{174,175} \mathrm{Hf}[40]$, and ${ }^{176} \mathrm{Hf}[41]$, and is associated with the ground-state configuration in ${ }^{177} \mathrm{Hf}[42]$. The neutron $[514] 7 / 2^{-}$orbital is predicted to exhibit a small signature splitting and both signatures should be populated with similar intensities. However, the signature partner for band MAB has not been identified in the present work.

\section{E. Bands HK1 and HK2}

Previously, bands HK1 and HK2 have been proposed to be associated with the $\pi[404] 7 / 2^{+}[402] 5 / 2^{+} \otimes \nu[633] 7 / 2^{+}$ and $\pi[404] 7 / 2^{+}[514] 9 / 2^{-} \otimes \nu[633] 7 / 2^{+}$intrinsic configurations, respectively. The suggestion was based on the energy systematics of isomers in the region and the properties of the bands, including the $\left(g_{K}-g_{R}\right) / Q_{0}$ ratios that were extracted from the low-spin levels in the bands [26, 27]. The two bands have now been extended to higher spins and, as a result, further insights in their configurations can be inferred. The $\sim 6 \hbar$ alignment gains in the two bands are associated with BC crossings, see Fig. 7(d). The crossing frequencies, $\hbar \omega \sim 320$ and 340 keV, are lower than the value $\hbar \omega \sim 380 \mathrm{keV}$ for the $\mathrm{BC}$ crossing in band $\mathrm{A}$. This effect is likely caused by the reduction of the neutron pairing correlations in the high- $K$ bands, as compared to that in band A, which is known to be configuration-dependent and related to the deformation of the bands [43]. All four signatures in the two bands should involve the A quasineutron because the AB crossing is blocked. Since orbitals a and b (based on the $\pi[404] 7 / 2^{+}$state) are almost degenerate, and orbitals c and $\mathrm{d}$ (based on $\pi[402] 5 / 2^{+}$) as well, it is not possible to determine which of the following configurations are more favorable in HK1: acA/bcA, adA/bdA, acA/adA, and bcA/bdA. For band HK2, on the other hand, the splitting between orbitals e an $\mathrm{f}$ (based on the $\pi[514] 9 / 2^{-}$state) is calculated to increase quickly for $\hbar \omega>300 \mathrm{keV}$, see Fig. 6. No signature splitting is observed in sequence HK2 up to the highest spins, suggesting that only one of the e and f orbitals is present in band HK2. The likely configurations for HK2 are afA/bfA for the positive/negative signatures, respectively.

The experimental $B(M 1) / B(E 2)$ ratios are further compared in Fig. 9 with the theoretical predictions. The detailed procedure for the calculations can be found, e.g., in our previous publication for ${ }^{168} \mathrm{Hf}$ [25]. For the collective gyromagnetic ratio $g_{R}$ and quadrupole moment $Q_{0}$ values of 0.35 and $5.7 \mathrm{~b}$ are used in the calculations, respectively, the latter is an average value measured for band A [21]. The intrinsic $g$-factors (with corresponding configurations given in parenthesis) are: 0.68 (ab), $1.5(\mathrm{~cd}), 1.26(\mathrm{ef}),-0.21(\mathrm{AB}),-0.21(\mathrm{CD})$. For the aligned BC neutron pair in band HK1, a measured total alignment of $6 \hbar$ is used, together with a zero contribution to the $K$ value. The experimental values and calculated ratios for the proposed configurations agree fairly well. The ratios for the two bands are clearly different from each other, indicating that the bands are associated with different quasiproton configurations. The suggested $\mathrm{f}$ quasiproton in HK2 has a larger alignment than the c (or d) quasiproton in HK1, consistent with the slightly larger alignment for HK2, as observed in Fig. 7(d). Thus, the observed band crossing, aligned angular momentum, and $B(M 1) / B(E 2)$ ratios further confirmed the previous configuration assignments for the high- $K$ bands.

\section{F. Band ED2 - evidence for a new band with enhanced deformation}

Band ED1 becomes yrast above spin $67 / 2^{+}$and has been interpreted as being built upon a second minimum in the potential energy surface $(\mathrm{PES})$ of ${ }^{171} \mathrm{Hf}$ at $\left(\varepsilon_{2}, \gamma\right)=\left(0.3,4^{\circ}\right)$, to be compared with the ND minimum at $\left(\varepsilon_{2}, \gamma\right)=\left(0.22,0^{\circ}\right)$. A configuration of $\pi\left(i_{13 / 2} h_{9 / 2}\right) \otimes \nu h_{9 / 2}$ has been proposed for band ED1, where the deeply down-sloping $i_{13 / 2}$ proton orbital drives the nucleus to an enhanced deformation relative to the ND structures [16]. Such an enhanced deformation has been confirmed by a quadrupole moment measurement [20]. Similar ED bands have been identified systematically in the neighboring isotopes ${ }^{168} \mathrm{Hf}[8],{ }^{170} \mathrm{Hf}[15]$, and ${ }^{175} \mathrm{Hf}[16]$.

The new band ED2 has not been linked to any known structure and, thus, the spins and energies of the levels remain unknown. The band is plotted in Figs. $7(\mathrm{~d})$ and $8(\mathrm{c})$ with an assumed spin of $53 / 2$ and an energy of 6880 $\mathrm{keV}$ for the lowest level. Therefore, the exact amount of alignment (the vertical position in Fig. $7(\mathrm{~d}))$ of the band is uncertain, but the alignment pattern is not affected. A noticeable feature is that band ED2, just as band ED1, exhibits neither a neutron nor a proton crossing up to the highest observed rotational frequency of $\hbar \omega \sim 640 \mathrm{keV}$. Such a frequency is well above $\hbar \omega \sim 500 \mathrm{keV}$, where proton crossings occur in all ND bands. This fact indicates that the proton orbitals are already occupied at lower frequencies in band ED2. The nearly flat dynamic moment of inertia $J^{(2)}$ of band ED2 as a function of $\hbar \omega$, as seen in Fig. 10, also indicates that no neutron or proton crossing 
occurs in the entire frequency range observed for this band. Therefore, band ED2 starts with a large initial aligned angular momentum at low spin. The high- $K$ bands also involve quasiprotons in their configurations for all levels but, like any band built on the normal deformed PES minimum, undergo neutron crossings for a rotational frequency $\hbar \omega$ between 250 and $500 \mathrm{keV}$. Band ED2 is clearly different from the high- $K$ bands, and is not associated with the normal deformed PES minimum.

It is likely that bands ED2 and ED1 have similar structures and are built upon the same minimum, the ED minimum. Band ED2 is more weakly populated than the ED1 sequence and does not extend as high in spin. This may be due to band ED2 lying at higher excitation energy and having a smaller alignment than band ED1. Based on these considerations, band ED2 is plotted in Fig. 8(c) with an assumed initial energy 200 keV above that of band ED1, and a slope smaller than that of ED1. It is also possible that band ED2 is the signature partner of band ED1, however, a search for interband transitions between the two sequences was not successful. Since band ED1 is built upon a PES minimum well separated from the ND minimum, and is associated with an intrinsic configuration very different from those of the ND structures, the de-excitation of its levels will proceed preferably within the band. As a result, the low-spin part of the band extends to very high energy above the yrast line before the decay occurs to the ND bands. The decay pathways are highly fragmented, as seen in Fig. 1. If band ED2 is indeed another band built on this ED minimum, similar to band ED1, such a decay mechanism will make it difficult to identify its decay pathways.

On the other hand, the possibility that band ED2 may correspond to a SD or TSD band has to be considered since such sequences have been observed in this mass region. It has been shown in our recent publication, and depicted in Fig. 10, that the values of dynamical moments of inertia $J^{(2)}$ behave differently for the observed SD bands and the ED bands in ${ }^{170-175} \mathrm{Hf}$ [16]. The values for SD bands decrease rapidly with rotational frequency, as represented by band SD1 of ${ }^{172} \mathrm{Hf}$. Such a rapid decrease is clearly absent in band ED2. The difference in the behavior of $J^{(2)}$ moments indicates that band ED2 probably does not belong to the group of SD bands. For ED bands, the $J^{(2)}$ values increase slightly or do not change much, as seen in band ED1 of ${ }^{171} \mathrm{Hf}$ whose values are similar to those of band ED2 at low frequencies and gradually increase at $\hbar \omega \sim 550 \mathrm{keV}$ due to a band crossing or an interaction with another band. The UC calculations also predicted a TSD minimum around $\left(\varepsilon_{2}, \gamma\right)=\left(0.43,20^{\circ}\right)$ for ${ }^{171} \mathrm{Hf}$, and the TSD band built upon this minimum lies $\sim 3 \mathrm{MeV}$ above the yrast line in the region of spins 20 to 40 [16]. The 43/2 level of band ED1 is $\sim 900 \mathrm{keV}$ above the yrast line, see Fig. 8(c), and is the observed state that has the highest excitation energy above the yrast line. From intensity considerations it is highly unlikely to observe the predicted TSD band even if it exists. Thus, band ED2 is likely a second band built upon the ED minimum, similar to band ED1. To ascertain the nature of the band, however, further experimental information, including the level spins and energies, is needed.

\section{SUMMARY}

High-spin properties of the nucleus ${ }^{171} \mathrm{Hf}$ were studied through the ${ }^{128} \mathrm{Te}\left({ }^{48} \mathrm{Ca}, 5 n\right)$ reaction. Previously known bands were extended to significantly higher spins and five new bands have been established. Spins and parities were assigned based on DCO ratio measurements. The intrinsic configurations of the bands were investigated, based on the observed neutron and proton crossings, on the results of cranking calculations performed with the UC code, and on systematic comparisons with data from neighboring nuclei. Specifically, bands H, E, and B have been extended to spins as high as $95 / 2^{-}, 89 / 2^{-}$, and $79 / 2^{+}$, respectively, so that the band crossings at $\hbar \omega \sim 500 \mathrm{keV}$ could be fully delineated. These crossings are proposed to be associated with excitations involving the $h_{11 / 2}, \alpha=-1 / 2$ (f) and $h_{9 / 2}, \alpha=+1 / 2$ (g) proton orbitals. Such mixed crossings have been established recently in the neighboring ${ }^{166,168,169}$ Hf. The onset of the proton crossing is observed in several other bands as well. Bands X2, X3, and X4 provided interesting details about various ways quasineutron alignments can occur and how exchange in character between bands proceeds in the lower-spin region, where the crossing between the one-quasineutron configuration A and the three-quasineutron configuration ABC occurs. Band ED2 exhibits an alignment pattern similar to that of band ED1. It is likely that band ED2 is the second band in ${ }^{171} \mathrm{Hf}$ built upon a second PES minimum which involves the proton $i_{13 / 2} \otimes h_{9 / 2}$ configuration and is associated with a deformation enhanced with respect to that of ND structures. Similar minima have been identified in several neighboring Hf isotopes and have been shown to coexist with ND minima and with the third (TSD) minimum in ${ }^{168} \mathrm{Hf}$. However, the decay pathways of band ED2 have not been established, and further experimental investigation is needed to ascertain the nature of this band.

\section{ACKNOWLEDGMENTS}

The authors wish to thank the ANL operation staff at Gammasphere. Special thanks also to J. P. Greene for target preparation. This work was supported by the U. S. Department of Energy, Office of Nuclear Physics, under grants 
DE-FG02-95ER40939 (MSU), DE-AC02-06CH11357 (ANL), DE-FG02-96ER40983 (UT), and DE-FG02-94ER40848 (UML), as well as by the National Science Foundation under grant PHY-0854815 (USNA).

[1] A. Bohr and B. R. Mottelson, Nuclear Structure, Vol. II, Benjamin, New York, 1975.

[2] S. Ødegård et al., Phys. Rev. Lett. 86, 5866 (2001).

[3] D. R. Jensen et al., Phys. Rev. Lett. 89, 142503 (2002).

[4] G. Schönwaßer et al., Phys. Lett. B552, 9 (2003).

[5] H. Amro et al., Phys. Lett. B553, 197 (2003).

[6] P. Bringel et al., Eur. Phys. J. A 24, 167 (2005).

[7] D. J. Hartley et al., Phys. Rev. C 80, 041304(R) (2009).

[8] R. B. Yadav et al., Phys. Rev. C 78, 044316 (2008).

[9] H. Amro et al., Phys. Lett. B506, 39 (2001).

[10] R. Bengtsson, www.matfys.lth.se/ ragnar/ultimate.html.

[11] T. Bengtsson, Nucl. Phys A496, 56 (1989).

[12] T. Bengtsson, Nucl. Phys A512, 124 (1990).

[13] H. Schnack-Petersen et al., Nucl. Phys. A594, 175 (1995).

[14] R. Bengtsson and H. Ryde, Eur. Phys. J. A 22, 355 (2004).

[15] A. Neußer-Neffgen et al., Phys. Rev. C 73, 034309 (2006).

[16] Y. C. Zhang et al., Phys. Rev. C 76, 064321 (2007).

[17] M. K. Djongolov et al., Phys. Lett. B560, 24 (2003).

[18] D. J. Hartley et al., Phys. Lett. B608, 31 (2005).

[19] D. T. Scholes et al., Phys. Rev. C 70, 054314 (2004).

[20] S. Mukhopadhyay et al., Phys. Rev. C 83, 044311 (2011).

[21] D. M. Cullen, et al., Nucl. Phys. A673, 3 (2000).

[22] I. Y. Lee, Nucl. Phys. A520, 641c (1990).

[23] D. C. Radford, Nucl. Instrum. Methods A 361, 297 (1995).

[24] K. S. Krane, R. M. Steffen, and R.M. Wheeler, Nucl. Data Tables 11 (1973) 351.

[25] R. B. Yadav et al., Phys. Rev. C. 80, 064306 (2009).

[26] G. D. Dracoulis and P. M. Walker, Nucl. Phys. A330, 186 (1979).

[27] D. M. Cullen, D. E. Appelbe, A. T. Reed, C. Baktash, and C.-H. Yu, Phys. Rev. C 55, 508 (1997).

[28] R. B. Firestone et al., Table of Isotopes, Vol. II (John Wiley \& Sons, 1996).

[29] D. R. Jensen et al., Eur. Phys. J. A 8, 165 (2000).

[30] K. A. Schmidt, Eur. Phys. J. A 12, 15 (2001).

[31] C.-H. Yu et al., Nucl. Phys. A511, 157 (1990).

[32] D. J. Hartley et al., Phys. Rev. C 72, 064325 (2005).

[33] E. M. Beck et al., Nucl. Phys. A464, 472 (1987).

[34] A. Fitzpatrick et al., Nucl. Phys. A582, 335 (1995).

[35] J. Recht et al., Nucl. Phys. A440, 366 (1985)

[36] D. J. Hartley et al., Phys. Rev. C 74, 054314 (2006).

[37] D. M. Cullen, et al., Nucl. Phys. A638, 662 (1998).

[38] G. B. Hegemann et al., Nucl. Phys. A618, 199 (1997).

[39] M. B. Smith et al., Eur. Phys. J. A 6, 37 (1999).

[40] N. L. Gjørup, P. M. Walker, G. Sletten, M. A. Bentley, B. Fabricius, and J. F. Sharpey-Schafer, Nucl. Phys. A582, 369 (1995).

[41] G. Mukherjee et al., Phys. Rev. C 82, 054316 (2010).

[42] S. M. Mullins, A. P. Byrne, G.D. Dracoulis, T. R. McGoram, and W. A. Seale, Phys. Rev. C 58, 831 (1998).

[43] J. D. Garrett, G. B. Hagemann, B. Herskind, J. Bacelar, R. Chapman, J. C. Lisle, J. N. Mo, A. Simcock, J. C. Willmott, Phys. Lett. B118, 297 (1982). 


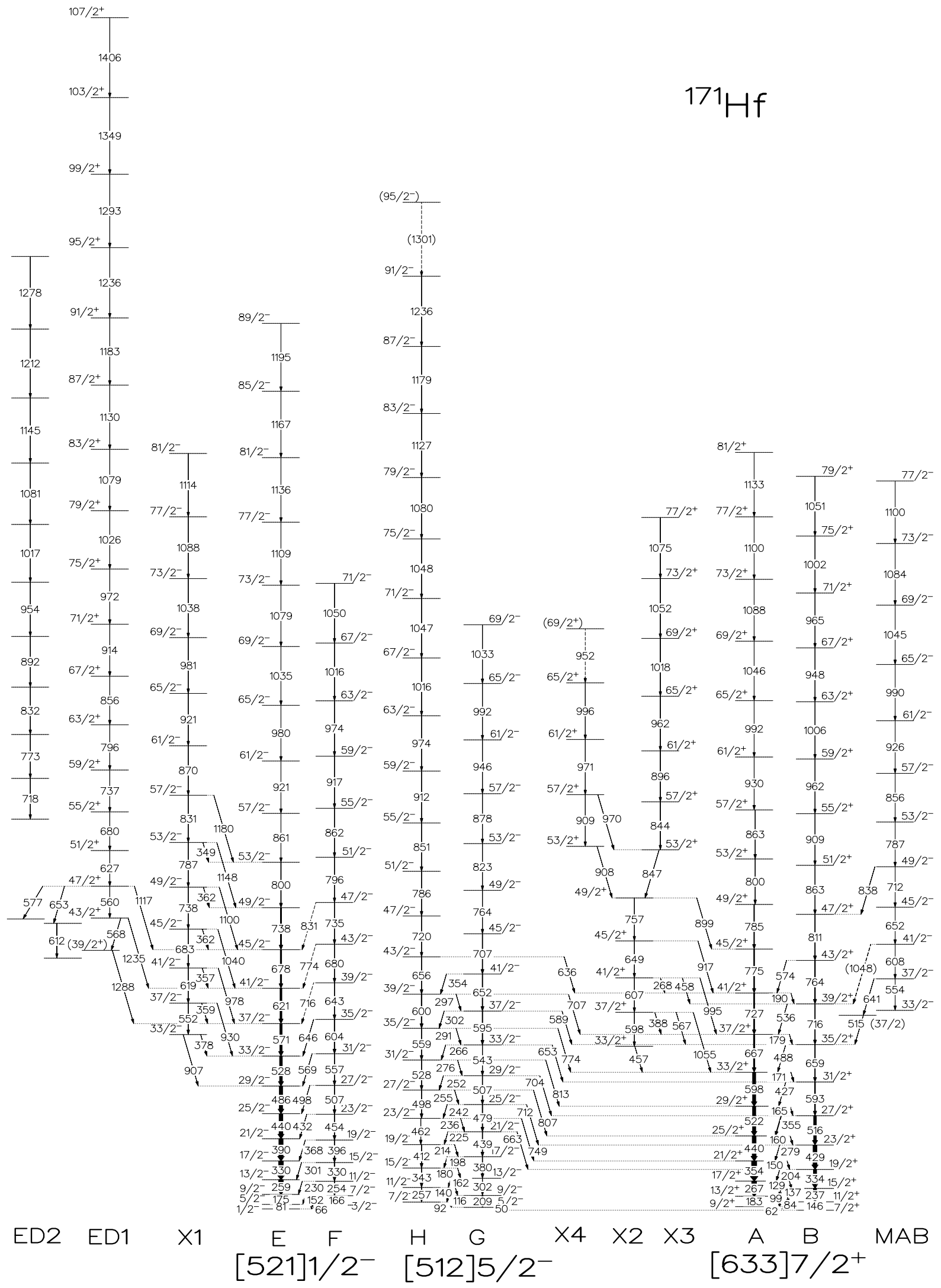

FIG. 1: Partial level scheme for ${ }^{171}$ Hf. Arrow widths are proportional to the relative intensities of the $\gamma$ rays. Energies are in $\mathrm{keV}$ and tentative transitions are shown with dashed lines. 


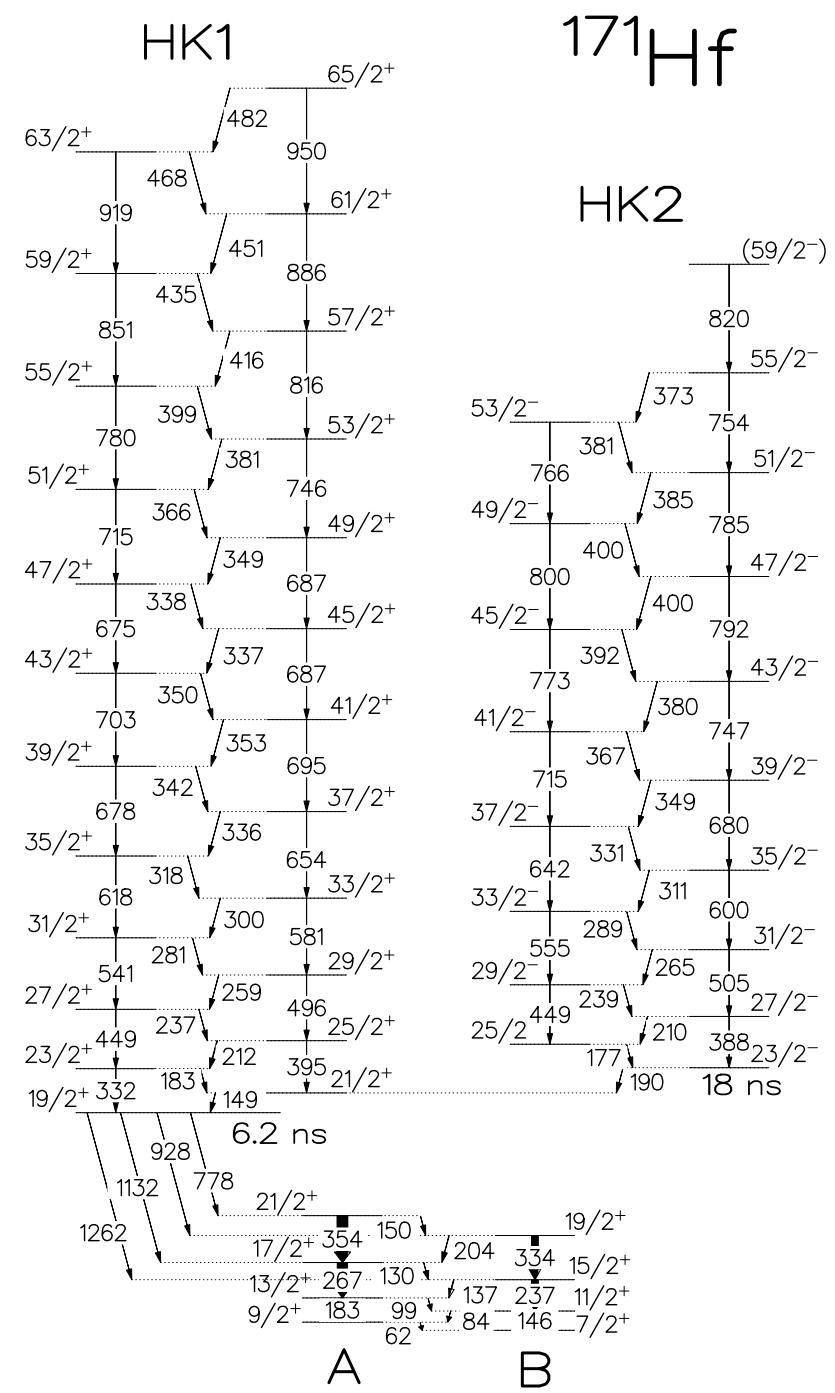

FIG. 2: Partial level scheme for ${ }^{171} \mathrm{Hf}$ displaying the high- $K$ bands. 

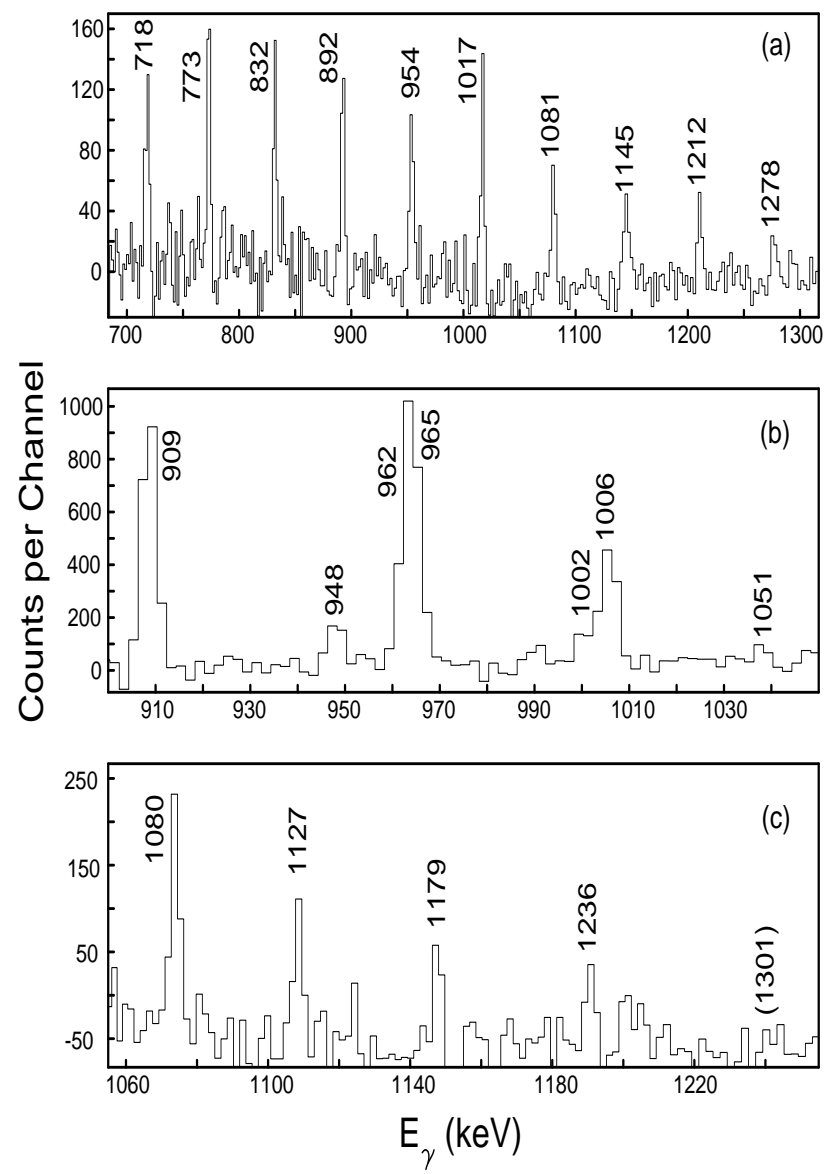

FIG. 3: Four-fold coincidence spectra for (a) band ED2, and the high-spin regions of (b) band B and (c) band H. The spectrum of band ED2 is a sum of triple coincidence gates on all band members. The spectra of bands B and $\mathrm{H}$ are sums of triple gates on transitions above levels $35 / 2^{+}$and $31 / 2^{-}$, respectively.

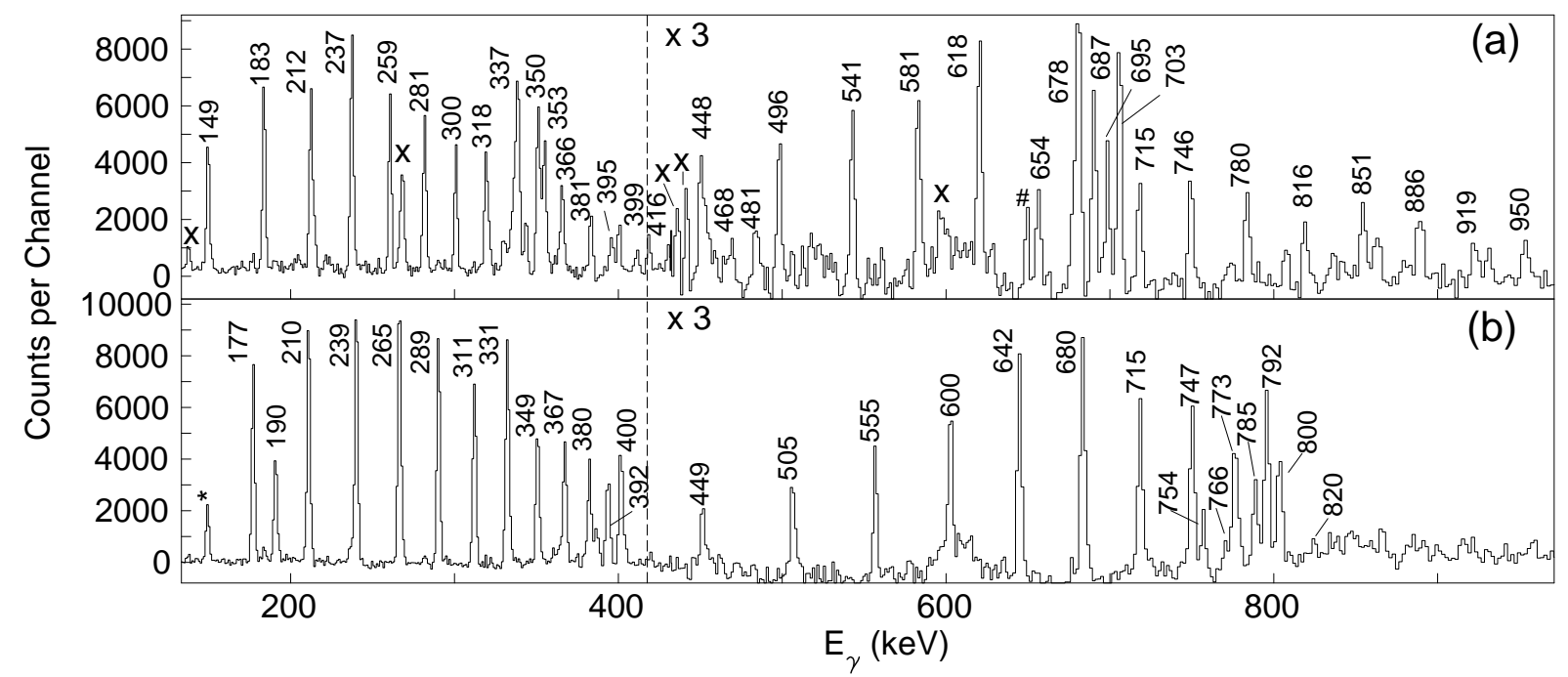

FIG. 4: Four-fold coincidence spectra for the high- $K$ bands, (a) band HK1 and (b) HK2, triple gated on the $M 1$ transitions in each band. The $M 1$ transitions are marked with plus signs ("+"), and the ND yrast transitions are marked with crosses ("X"). The "\#" symbols in the HK1 spectrum denote contaminations from neighboring nuclei. The star symbol ("*") in the HK2 spectrum denotes the $149-\mathrm{keV}$ peak which is the lowest $M 1$ transition in band HK1. 


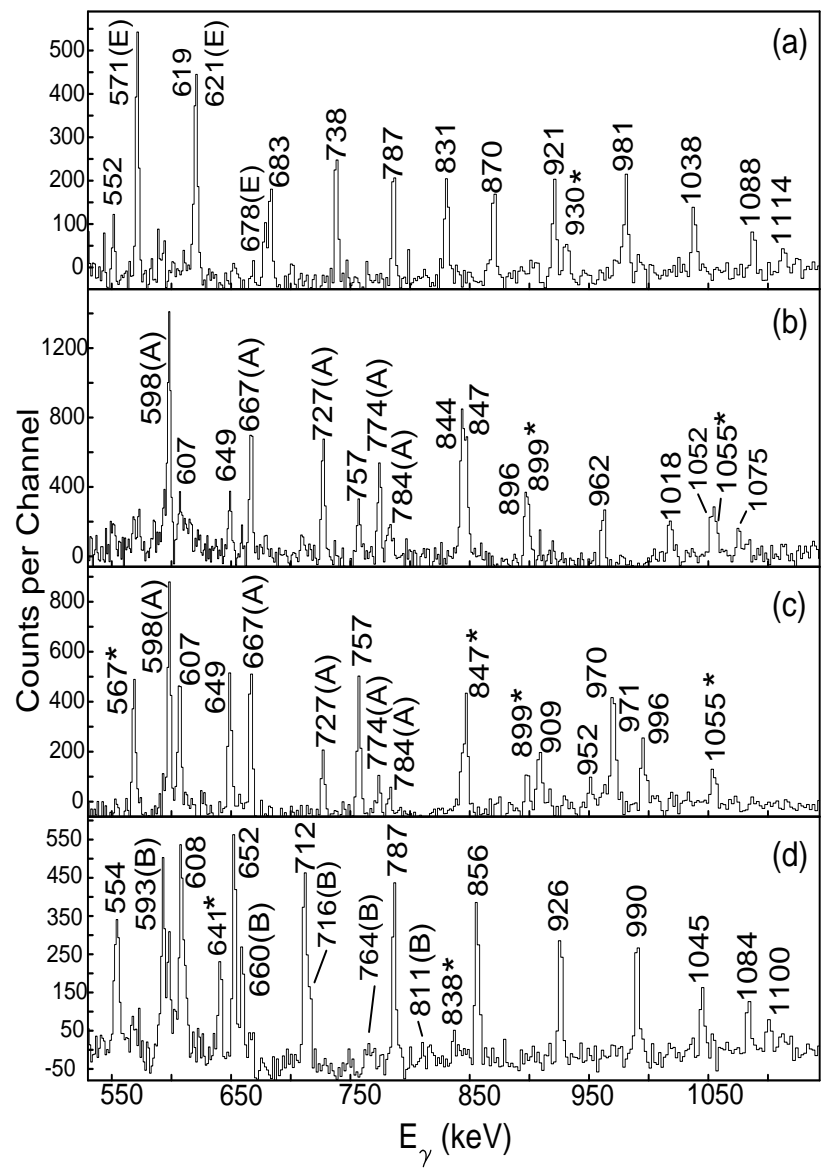

FIG. 5: Representative four-fold coincidence spectra for the high-spin regions of bands (a) X1, (b) X2+X3, (c) X2+X4, and (d) MAB. The star symbols ("*”) denote the interband linking transitions. Spectra of bands X1 and MAB are sums of triple gates on all band members. The spectrum of bands X2+X3 is a sum of triple gates on all transitions in band X3. The spectrum of bands $\mathrm{X} 2+\mathrm{X} 4$ is a sum of coincidence gates between transitions in band X2 and X4. 

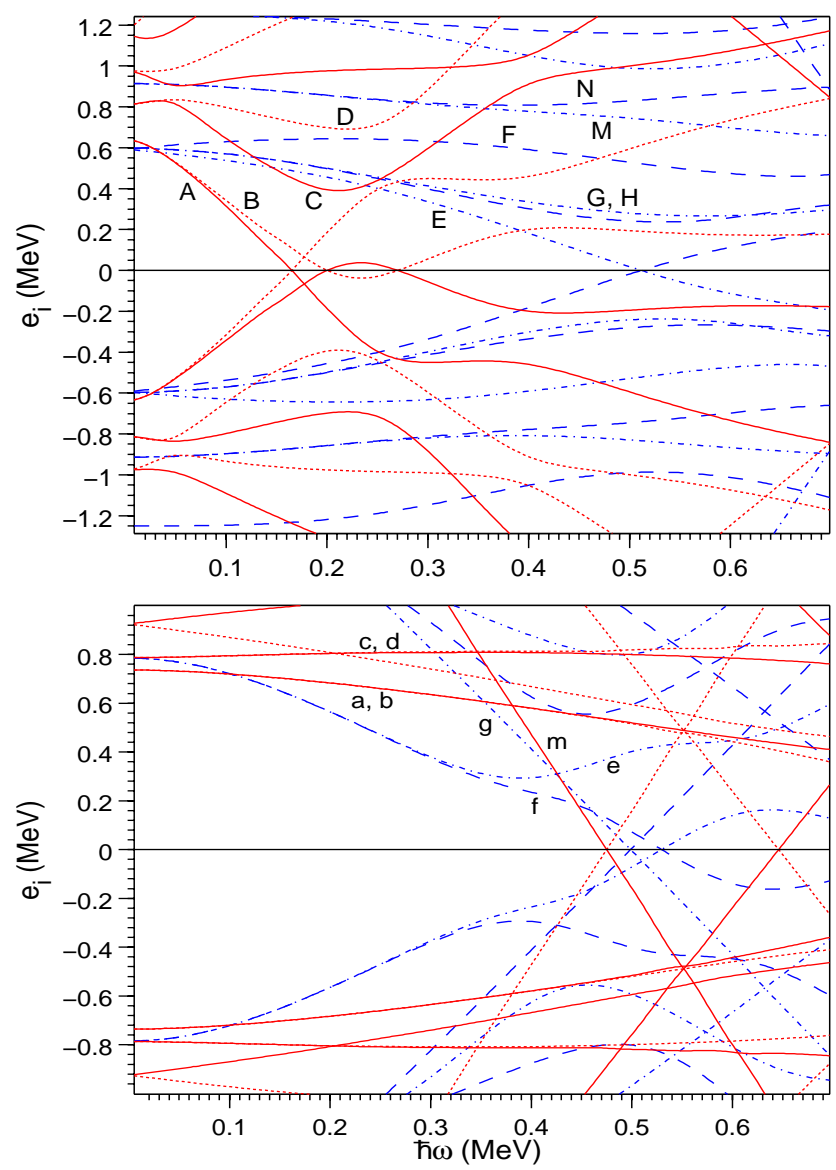

FIG. 6: (Color online) Quasiparticle diagrams for neutrons (upper panel)) and protons (lower panel) for ${ }^{171} \mathrm{Hf}$ calculated at $\epsilon_{2}=0.225, \epsilon_{4}=0.03$, and $\gamma=0^{\circ}$. The levels are labeled by parity and signature as $(+,+1 / 2)$ solid lines, $(+,-1 / 2)$ dotted lines, $(-,+1 / 2)$ dot-dashed lines, and $(-,-1 / 2)$ dashed lines. 


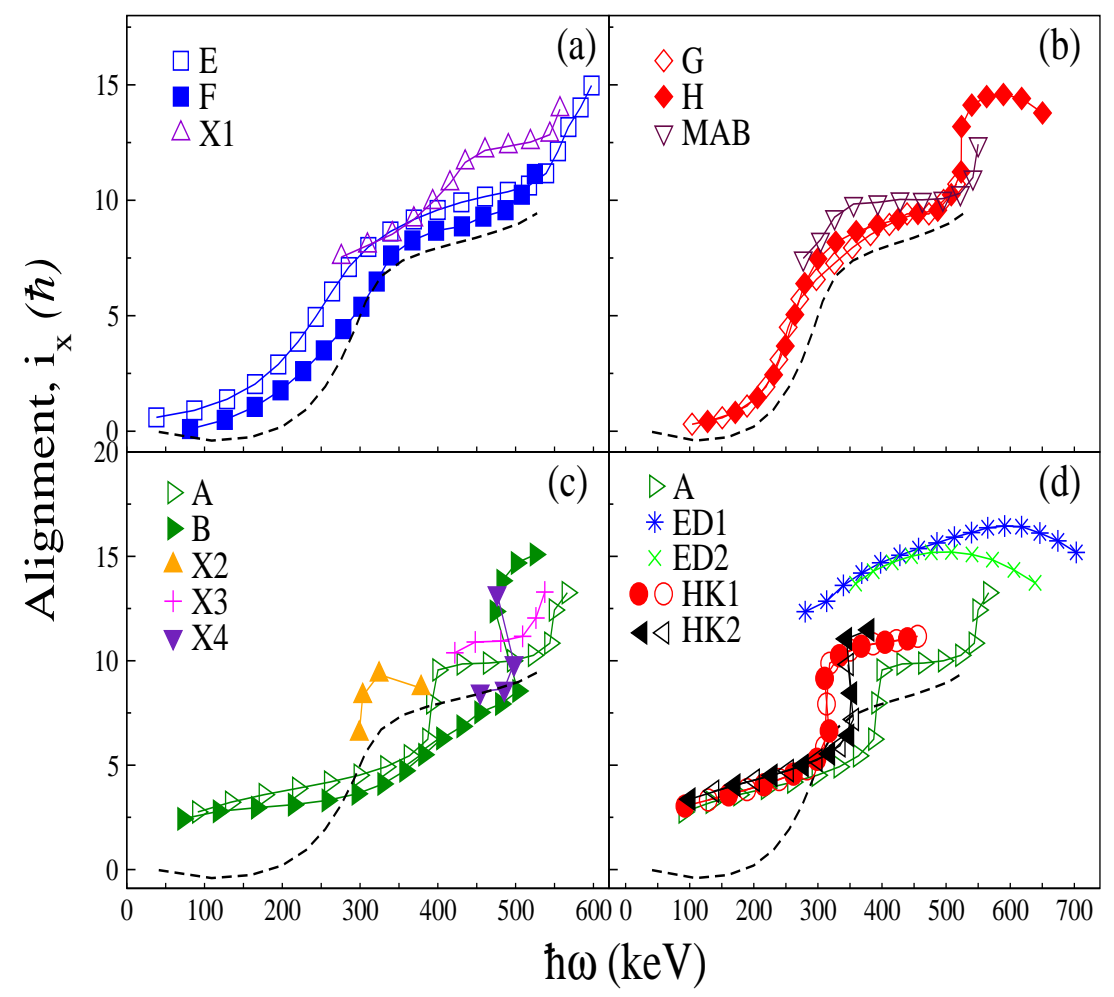

FIG. 7: (Color online) Alignments for the bands in ${ }^{171} \mathrm{Hf}$. The dashed line represents the alignment observed in the ground-state band in ${ }^{170}$ Hf. The plot of band ED2 is based on assumed spin values, see text for details.
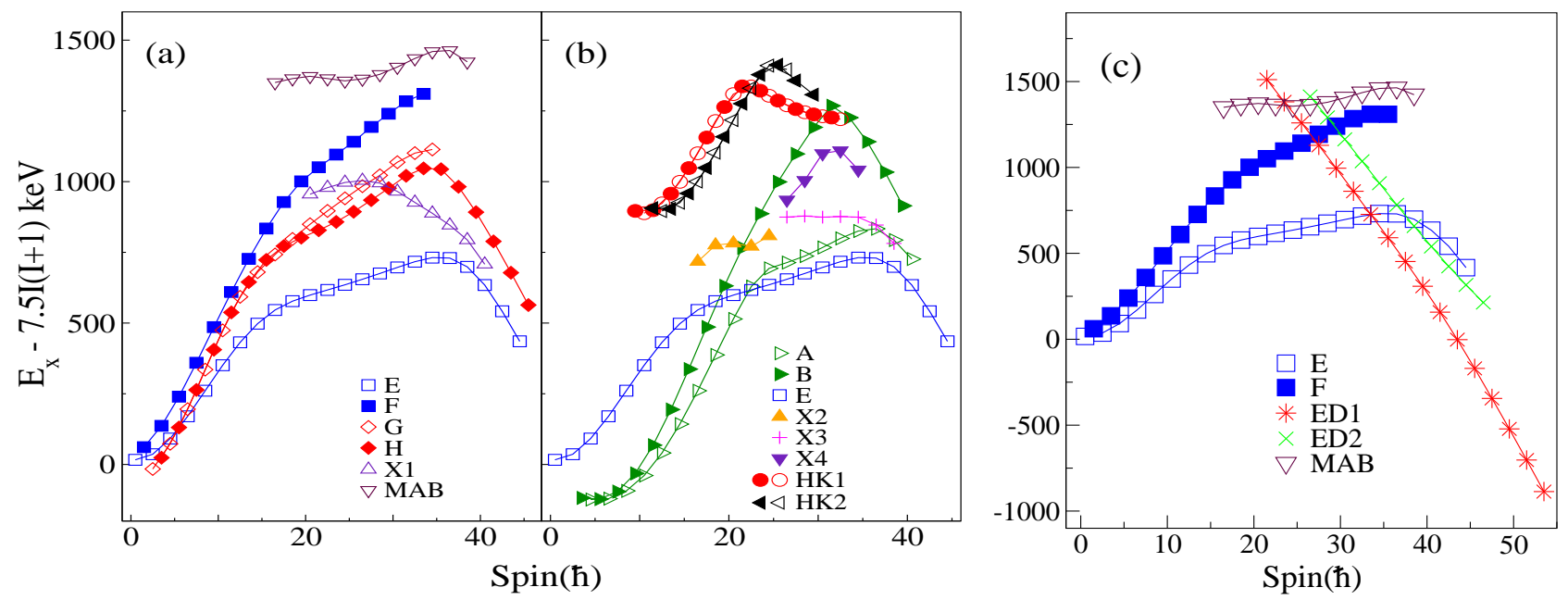

FIG. 8: (Color online) Excitation energies, minus a rigid-rotor reference, as a function of spin for the bands in ${ }^{171} \mathrm{Hf}$. The plot of band ED2 is based on assumed excitation energy and spin values, see text for details. 


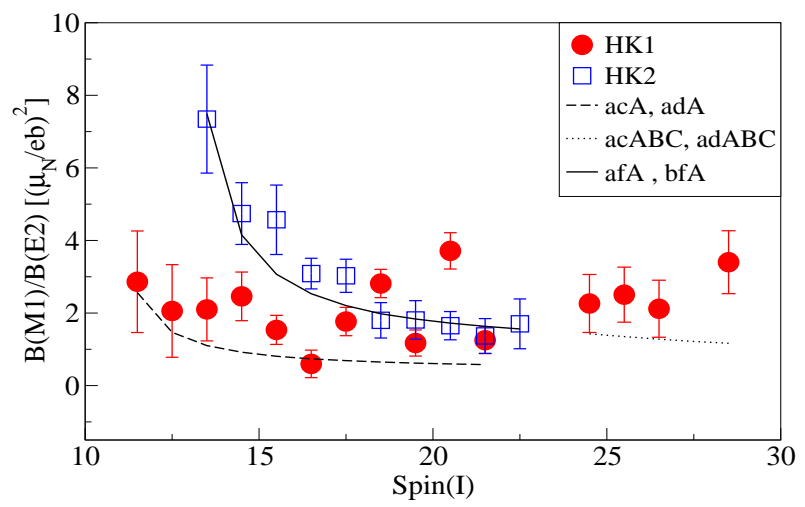

FIG. 9: (Color online) Experimental B(M1)/B(E2) ratios (data points) compared to theoretical values (lines) for bands HK1 and HK2.

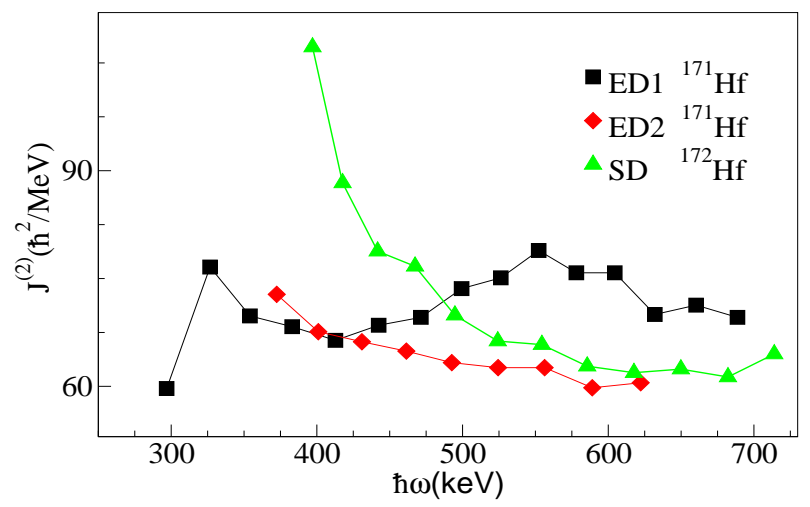

FIG. 10: (Color online) Dynamic moments of inertia $J^{(2)}$ as a function of rotational frequency for bands ED1 and ED2 in ${ }^{171} \mathrm{Hf}$ and band SD1 in ${ }^{172} \mathrm{Hf}[16]$. 